\title{
1 Normal spindle positioning in the absence of EBPs and dynein plus-end
}

\section{2 tracking in C. elegans}

4 Ruben Schmidt ${ }^{1,2}$, Anna Akhmanova ${ }^{1}$ and Sander van den Heuvel ${ }^{2 *}$

$7{ }^{1}$ Cell Biology, Faculty of Sciences, Department of Biology, Utrecht University,

8 Padualaan 8, $3584 \mathrm{CH}$ Utrecht, The Netherlands.

9

$10{ }^{2}$ Developmental Biology, Faculty of Sciences, Department of Biology, Utrecht

11 University, Padualaan 8, 3584 CH Utrecht, The Netherlands.

12

13 Short title: Two independent dynein populations at the cortex

14

$15{ }^{*}$ Correspondence and requests for materials should be addressed to S.v.d.H. (email:

16 s.j.l.vandenHeuvel@uu.nl; tel. +31 30253 3573) or A.A. (a.akhmanova@uu.nl; tel.

$17+31302532328)$. 


\section{Abstract}

2

3 The position of the mitotic spindle is tightly controlled in animal cells, as it

4 determines the plane and orientation of cell division. Interactions between

5 cytoplasmic dynein at the cortex and astral microtubules generate pulling forces

6 that position the spindle. In yeast, dynein is actively delivered to the cortex

7 through microtubule plus-end tracking complexes. In animal cells, an

8 evolutionarily conserved Ga-GPR-1/2 $2^{\text {Pins/LGN-LIN-5 }} 5^{\text {NuMA }}$ cortical complex interacts

9 with dynein and is required to generate pulling forces, but the mechanism of

10 dynein recruitment to the cortex is unclear. Using CRISPR/Cas9-assisted

11 recombineering, we fluorescently labeled endogenous DHC-1 dynein in C. elegans.

12 We observed strong dynein plus-end tracking, which depended on the end-

13 binding protein EBP-2. Complete removal of the EBP family abolished dynein plus-

14 end tracking but not LIN-5-dependent cortical localization. The ebp-1/2/3 deletion

15 mutant, which was viable and fertile, showed increased cortical microtubule

16 retention; however, pulling forces and spindle positioning were normal. These data

17 indicate that dynein recruited from the cytoplasm creates robust pulling forces. 
1 The mitotic spindle determines the plane of cell cleavage through its position and

2 interactions with the cell cortex (reviewed in refs. ${ }^{1,2}$ ). The position of the spindle

3 thereby controls the relative size and location of daughter cells formed during cell

4 division. In addition, dependent on the position of the mitotic spindle, polarized

5 cells may divide either symmetrically or asymmetrically. Asymmetric cell divisions

6 generate cell diversity and allow long-term retention of somatic stem cells during

7 the development of organisms and maintenance of tissues. Thus, accurate

8 positioning of the spindle is critical for a wide range of processes that include the

9 formation of gametes, integrity of tissues, creation of different cell types, and

10 coordination of stem cell proliferation and differentiation.

11 The C. elegans early embryo provides an important in vivo model for studies

12 of regulated spindle positioning. The one-cell embryo divides asymmetrically

13 based on an anterior-posterior (A-P) polarity axis established after fertilization ${ }^{3}$.

14 Classical spindle severing experiments revealed that pulling forces acting from the

15 cell cortex on astral microtubules (MTs) position the spindle, and that these forces

16 are higher in the posterior than in the anterior ${ }^{4}$. This asymmetry in forces leads to

17 displacement of the spindle towards the posterior, and allows cell cleavage to

18 create two blastomeres of unequal size and developmental fate ${ }^{5}$.

Genetic screens and biochemical experiments have revealed a variety of

21 factors important for the generation and distribution of mitotic pulling forces.

22 Among these are the evolutionarily conserved proteins $G \alpha, G P R-1 / 2^{\text {PINS/LGN }}$ and LIN-

$235^{\text {Mud/NuMA6-10 }}$. These proteins form a complex known as the force generator complex 
1 (FGC), which is regulated downstream of A-P polarity, thus bridging cell polarity

2 and mitotic force generation. Cortical pulling forces also depend on cytoplasmic

3 dynein ${ }^{11}$. This large multi-subunit protein complex is the major minus-end directed

4 motor in the cell and is essential for a large variety of cellular processes, including

5 bipolar spindle assembly, mitotic checkpoint regulation, centrosome positioning,

6 and intracellular transport (reviewed in ref. ${ }^{12}$ ). In the current model, the FGC

7 recruits dynein to the cell cortex either directly or indirectly via an extended N-

8 terminal region of LIN-5 $5^{13}$. Dynein subsequently associates with astral MTs in an

9 end-on configuration, inducing pulling forces via both MT depolymerization and

10 minus-end directed movement. Both in vivo and in vitro studies support this

11 model ${ }^{11,14-16}$.

It remains unclear, however, how dynein is recruited to the cortex and how

13 the dynamic instability of MTs is coupled to force generation and mitotic spindle

14 positioning ${ }^{17}$. MT growth and shrinkage are spatiotemporally modulated by a large

15 variety of MT-associated proteins (MAPs), a subset of which concentrates at the

16 growing MT plus-end (reviewed in ref. ${ }^{18}$ ). These MT plus-end-tracking proteins

17 (+TIPs) form a highly interconnected and dynamic network, which is regulated in a

18 cell cycle and position-dependent manner to fine-tune MT dynamics ${ }^{19-22}$. Members

19 of the end-binding (EB) protein family are seen as master regulators of the +TIP

20 network, as they bind autonomously to the growing MT end and recruit multiple

21 other + TIPs $^{19,23-25}$ (reviewed in ref. ${ }^{18}$ ).

Interestingly, dynein is known to behave as a +TIP in a variety of cellular

23 contexts ${ }^{26-29}$. Dynein plus-end tracking in mammals is classically regarded as a 
1 'search-and-capture' mechanism, by which the complex finds its cargo molecules

2 via the MT plus-end before initiating minus-end directed transport ${ }^{30,31}$. In the

3 budding yeast S. cerevisiae, dynein plus-end tracking appears coupled to off-

4 loading at the cortex and association of dynein with its cortical anchor Num132-34.

5 Disruption of dynein plus-end recruitment is thus associated with spindle

6 positioning defects in yeast. Interactions between EB1, CLIP-170, and the dynactin

7 protein p150Glued also recruit the human dynein complex to the MT plus-end ${ }^{35-37}$.

8 However, whether dynein plus-end tracking is generally involved in spindle

9 positioning remains to be determined.

In this study, we explore the localization of dynein during mitotic pulling

11 force generation in the one-cell C. elegans embryo. Using CRISPR/Cas9-mediated

12 genome editing ${ }^{38-42}$, we labeled the endogenous dynein complex and studied its

13 dynamic localization during mitosis using a variety of techniques. We observed two

14 populations of dynein at the cortex: an EB-protein dependent MT plus-end tracking

15 dynein population and a LIN-5-dependent MT independent population. By

16 generating single, double and triple EB protein family gene knock-out mutants, we

17 show that spindle positioning and embryonic development can proceed as normal

18 in the absence of dynein plus-end tracking, and despite alterations of MT

19 dynamics. 


\section{Results}

\section{Visualization of the endogenous dynein complex}

4 In order to visualize the dynamics of the dynein complex in the early C. elegans

5 embryo we applied CRISPR/Cas9-mediated genome editing. We decided to target

6 the dynein heavy chain as opposed to an accessory subunit in order to label all

7 possible compositions of the dynein complex. In C. elegans the dynein heavy chain

8 is encoded by dhc-1, which has been labeled with fluorescent proteins (FPs) at both

9 its $\mathrm{N}$ - and C-terminus in different systems ${ }^{43-45}$, resulting in targeting of the DHC-1

10 tail and motor domain, respectively (Fig. 1A). To allow for functional comparison,

11 we explored both strategies by inserting mcherry directly upstream of the dhc-1

12 stop codon, and mcherry or egfp directly upstream of the dhc-1 start codon. A linker

13 region encoding 4 glycine residues was always inserted between FPs and $d h c-1$ to

14 preserve independent protein folding (Fig. 1A).

16 We obtained multiple homozygous viable knock-in strains using both strategies.

17 However, C-terminal tagging of $d h c-1$ resulted in both severe embryonic lethality

$18(72,5 \% \pm 9,2$ vs. $0,2 \% \pm 0,3$ in N2), and a strong reduction in brood size $(30,0 \pm 18,4$

19 animals vs. $323,3 \pm 15,5$ in N2) (Fig. 1B). Observation of early embryos by

20 differential interference contrast (DIC) microscopy revealed spindle positioning

21 and cell division defects from the one-cell stage onward. These phenotypes are in

22 accordance with perturbed dynein function ${ }^{46}$, which we confirmed in two

23 independent strains. This led us to conclude that C-terminal tagging of DHC-1 
1 causes a partial loss of DHC-1 function, as a complete loss of function would not be

2 homozygous viable. We did not further study dynein dynamics using these strains.

4 In contrast, animals with homozygous N-terminally tagged DHC-1 were fully viable

$5 \quad(0,1 \pm 0,2$ in mcherry:::dhc- 1 vs. $0,5 \pm 0,4$ in egfp:::dhc- 1 vs. $0,2 \% \pm 0,3$ in N2), and

6 showed normal brood size $(325,0 \pm 38,8$ in mcherry:: dhc- 1 vs. $318,8 \pm 34,2$ in

7 egfp::dhc-1 vs. $323,3 \pm 15,5$ in N2) (Fig. 1B). Worms appeared healthy during all

8 stages of development, and we observed no obvious abnormalities in early

9 embryonic development for either of these strains by DIC microscopy. From these

10 data we conclude that N-terminal tagging of DHC-1 with either mCherry or eGFP

11 does not perturb dynein function.

13 Observation of adult worms by wide-field fluorescence microscopy revealed

14 expression of both mCherry::DHC-1 and eGFP::DHC-1 in all somatic tissues of the

15 worm. Notably, DHC-1 is enriched in the germ-line, which is known for the robust

16 silencing of transgenes (Fig. 1C, blow-up). Next we followed early embryos by time-

17 lapse spinning disc confocal laser microscopy (SDCLM). A diffuse cytoplasmic pool

18 of DHC-1 was present in the cytoplasm during all stages of the cell cycle; in

19 addition, DHC-1 was located at the nuclear envelope, centrosomes, kinetochores,

20 kinetochore MTs, central spindle, astral MTs and the cell cortex during mitosis (Fig.

21 1D). This localization pattern is in accordance with earlier results from

22 immunohistochemistry and transgene overexpression experiments ${ }^{11,43,46,47}$. In

23 addition, we also noticed comet-like accumulations of dynein radiating from the 
1 centrosomes to the cell periphery in a pattern that appeared to follow the astral MT

2 network during metaphase and anaphase.

4 Dynein tracks MT plus-ends during mitosis

5 To further explore this localization pattern, we co-expressed Ppie-1-driven gfp::ß-

6 tubulin ${ }^{48}$ with mcherry::dhc-1 and imaged embryos by simultaneous dual-color

7 time-lapse SDCLM. We observed that dynein comets were MT-associated, most

8 likely concentrating at the growing plus-end (Fig. 2A, arrow, Supplementary Movie

9 1). Since dynein is a minus-end directed motor protein, we reasoned that cortex-

10 directed dynein comets most likely revealed a plus-end tracking population. To test

11 this possibility, we co-expressed Ppie-1-driven ebp-2::gfp as a germ-line MT plus-

12 end marker ${ }^{49,50}$ with mcherry:: $d h c-1$. This revealed strong co-localization of dynein

13 and EBP-2::GFP comets (Fig. 2B, Supplementary Movie 2). Intensity profile analysis

14 of both mCherry::DHC-1 and EBP-2::GFP comets shows strong similarity between

15 the two, indicating that plus-end tracking of dynein is strongly similar to that of

16 EBP-2 (Fig. 2C). Next, we measured the cortex-directed velocity of mCherry::DHC-1

17 and EBP-2::GFP comets. Measurements indicated that comet velocities are almost

18 identical $(0,74 \pm 0,11 \mu \mathrm{m} / \mathrm{s}$ for EBP-2::GFP $(\mathrm{N}=116)$ and $0,71 \pm 0,13 \mu \mathrm{m} / \mathrm{s}$ for

19 mCherry::DHC-1 comets $(\mathrm{N}=57), \mathrm{p}=0,136)$ (Fig. 2D). These results are in line with

20 earlier quantifications of MT growth speeds during metaphase $\left(0.72 \pm 0.02 \mu \mathrm{m} / \mathrm{s}^{49}\right)$.

21 In order to confirm that DHC-1 plus-end tracking is not an artefact due to protein

22 tagging, and to find out whether other components of the dynein complex are

23 present at the MT plus-end, we also studied the localization of dynactin. This 
1 protein complex is required for a broad range of dynein functions ${ }^{12}$, one of which is

2 spindle positioning in C. elegans ${ }^{51}$. Both GFP::DNC-1 p150glued $^{\text {and GFP::DNC-2 }}$ p50/dynamitin

3 showed clear overlap with mCherry::DHC-1 comets (Supplementary Fig. 1). Thus,

4 we conclude that the dynein comets we observe represent plus-end tracking of the

5 endogenous dynein complex, with high similarity in localization to EB plus-end

6 tracking.

8 An important question is how MT plus-end tracking of the dynein complex relates

9 to pulling force generation. Thus, we studied cortical dynein localization in mitosis by dual-color total internal reflection fluorescence (TIRF) microscopy. We observed

11 the simultaneous appearance of dynein comets with end-on MT-cortex contacts at

12 the cortex (Fig. 2E, Supplementary Movie 3). Interestingly, when MTs stopped

13 growing (as judged by loss of EBP-2::GFP signal), we also observed loss of the

14 concentrated mCherry::DHC-1 signal at the cortex (Fig. 2F, Supplementary Movie

15 4). This indicates that most dynein is lost from the plus-end when the MT switches

16 from a growing to a shrinking state.

17 To explore the functional relevance of dynein plus-end tracking to spindle

18 positioning, we imaged dynein dynamics during cortical force generation. Single

19 force generation events can be visualized by invaginations of the plasma

20 membrane ${ }^{52}$. To visualize membrane invaginations, we used CRISPR/Cas9-

21 mediated genome editing to generate a strain expressing $\mathrm{PH}::$ eGFP driven by Peft-3

22 in all tissues including the germ-line. This transgene was integrated in the

23 CXTi10816 locus on chromosome IV previously used to achieve germ-line 
1 expression of single-copy integrated transgenes ${ }^{53}$. Interestingly, dual-color imaging

2 of PH::eGFP and mCherry::DHC-1 reveals strong co-occurrence of dynein comets

3 reaching the cortex and the formation of membrane invaginations in 13/25 events

4 (Fig. 2G, Supplementary Movie 5). However, we could not detect enrichment of

5 mCherry::DHC-1 after the initiation of membrane invaginations. This indicates that

6 a significant part of the dynein population present in a comet at the MT plus-end

7 dissipates upon membrane contact and is most likely not involved in the

8 generation of cortical pulling forces.

\section{Dynein plus-end tracking and $e b p-1 / 2 / 3$ are not required for spindle}

\section{1 positioning and development}

12 Although disappearing during membrane invagination, it remained unclear

13 whether the MT plus-end tracking population of dynein contributes to cortical

14 pulling force generation. To investigate this possibility, we sought to specifically

15 disrupt dynein plus-end accumulation by removing +TIPs. To date, MT plus-end

16 tracking has not been thoroughly explored in C. elegans, though two homologs of

17 the mammalian EBs, EBP-1 and EBP-2, have been shown to exhibit plus-end

18 tracking activity when overexpressed in the embryo ${ }^{49}$. An annotated third

19 homolog, ebp-3, appears to have arisen by a duplication of the genomic region

20 containing the ebp-1 gene. This duplication likely occurred relatively recently, as

21 judged by the almost complete sequence identity between ebp-1 and ebp-3.

22 However, alignment of the predicted protein sequences revealed that EBP-3 lacks

23 the calponin homology $(\mathrm{CH})$ domain, which is required for recognition of the MT 
1 plus-end ${ }^{24,54}$. Thus, we suspect that ebp-3 is a pseudogene. Because of the high

2 sequence similarity between ebp-1 and ebp-3, we refer to these genes collectively

3 as ebp-1/3.

4

5 We knocked down expression of $e b p-1 / 3$ and $e b p-2$ by RNAi, and imaged one-cell

6 egfp:: dhc-1 embryos during mitosis by time-lapse confocal microscopy. While loss

7 of ebp-1/3 expression did not appear to affect eGFP::DHC-1 plus-end tracking, loss

8 of $e b p-2$ expression abolished the appearance of dynein comets during mitosis

9 (Fig. 3A). Interestingly, this did not result in obvious spindle positioning or cell

10 division defects upon initial inspection.

11 To further explore the role of the EBPs and dynein plus-end tracking during

12 mitosis, and to circumvent the possibility of incomplete RNAi knockdown, we

13 generated knock-out alleles for both ebp-2 and ebp-1/3 (Fig. 3B). Making use of

14 CRISPR/Cas9, the neighboring ebp-1 and ebp-3 genes were removed together, by

15 excising a $\sim 17 \mathrm{~kb}$ region (referred to as $\Delta \mathrm{e} b p-1 / 3$ ). The $e b p-2$ knock-out allele lacks

16 both the coding region as well as $\sim 1 \mathrm{~kb}$ upstream of the start codon (referred to as

$17 \Delta e b p-2)$. Both the $\Delta e b p-1 / 3$ and $\Delta e b p-2$ strains could be stably maintained as

18 homozygotes, indicating that neither ebp-1/3 nor ebp-2 are strictly required for

19 normal development and reproduction.

20 When crossed with egfp::dhc-1, we observed effects on dynein localization

21 identical to those of the RNAi experiments. Dynein still localized to the MT plus-end

22 in $\Delta e b p-1 / 3$; egfp:: $d h c-1$ embryos, while this localization was completely absent in

23 the $\Delta e b p$-2; egfp::dhc-1 early embryos (Fig. 3C, Supplementary Movie 6). At the 
1 same time, dynein localization to the nuclear envelope, spindle midzone and poles

2 did not appear to be affected in either case, which indicates that dynein depends

3 on EBP-2 specifically for recruitment to astral MT plus-ends (Supplementary Fig. 2).

4

5 While generally showing no defects in development, $\Delta e b p-1 / 3$ caused a slight but

6 statistically significant reduction in brood size $(312,50 \pm 42,53$ in $\Delta e b p-1 / 3$ vs.

$7329,75 \pm 35,11$ in N2), while $\Delta e b p-2$ did not $(335,25 \pm 28,04$ in $\Delta e b p-2$ vs. $329,75 \pm$

35,11 in N2). In addition, $\Delta e b p-1 / 3$, but not $\Delta e b p-2$, displayed a significant but biologically probably irrelevant increase in embryonic lethality $(0,47 \% \pm 0,16$ in $\Delta e b p-1 / 3$ and $0,00 \% \pm 0,00$ in $\Delta e b p-2$ vs. $0,07 \% \pm 0,14$ in N2) (Fig. 3D). In order to assess whether development and reproduction could proceed in the absence of all three EBPs, we crossed $\Delta e b p-1 / 3$ with $\Delta e b p$-2 to generate the triple knock-out mutant $\Delta \mathrm{e} b p-1 / 2 / 3$. Interestingly, this combination was viable and could be maintained as triple homozygous animals. The triple mutant showed a reduction in brood size $(240,38 \pm 62,45$ vs. $329,75 \pm 35,11$ in N2), which is stronger than the observed reduction in $\Delta e b p-1 / 3$ animals and indicates some redundancy amongst $e b p-1 / 3$ and ebp-2. Embryonic lethality remained low $(1,79 \% \pm 1,96$ vs. 0,07\% $\pm 0,14$ in N2), which is remarkable given the complete absence of EBPs and expected profound disruption of the +TIP network. While $\Delta e b p-1 / 2 / 3$ worms generally show no developmental defects, we did observe a partly penetrant pleiotropic phenotype amongst adult worms. This included low frequencies of dumpy, sterile, and/or uncoordinated animals, as well as lethal larvae that exploded through the vulva. In addition, some triple mutant worms appeared to form small bulges in the 
1 skin. Because of the low penetrance and unpredictable nature, we did not further

2 examine these abnormalities.

4 In order to assess whether spindle positioning was affected in the $\Delta e b p$ mutants,

5 we followed early embryos by DIC time-lapse microscopy and characterized key

6 mitotic events. Importantly, asymmetric positioning of the spindle and subsequent

7 asymmetric division of the one-cell embryo was not affected in $\Delta e b p-1 / 3, \Delta e b p-2$ or

$8 \Delta e b p-1 / 2 / 3$ embryos (Supplementary Fig. 3A). $\Delta e b p-1 / 2 / 3$ embryos did exhibit a

9 slight change in geometry, as they attained a rounder shape compared to wild-

10 type (wt) embryos (Supplementary Fig. 3B). The position of pronuclear meeting

11 along the A-P axis of the embryo did not change significantly (Supplementary Fig.

$123 \mathrm{C}$ ), while centration of the nucleocentrosomal complex occurred slightly more

13 posterior in $\Delta e b p-1 / 3, \Delta e b p-2$ and $\Delta e b p-1 / 2 / 3$ embryos. The angle at which the

14 metaphase spindle is set up did not change significantly (Supplementary Fig. 3E),

15 while elongation of the anaphase spindle was slightly increased only in the $\Delta e b p$ -

$16 \quad 1 / 2 / 3$ mutant (Supplementary Fig. 3F). Regardless of these very small variations in

17 mitotic events, they did not appear to have any significant impact on the outcome

18 of mitosis. However, all $\Delta e b p$ strains did exhibit an accelerated progression through

19 mitosis compared to wt embryos (Fig. 3E and Supplementary Fig. 4A,

20 Supplementary Movie 7). This effect occurred in $\Delta e b p-1 / 3$ and $\Delta e b p-2$ mutants, but

21 was most dramatic in $\Delta \mathrm{ebp}-1 / 2 / 3$. Both the time to progress from pronuclear

22 meeting to nuclear envelope breakdown (NEBD) (Supplementary Fig. 4B), as well as

23 from the start of chromosome segregation until completion of furrow ingression 
1 was significantly decreased (Supplementary Fig. 4D). We do not know the

2 mechanism behind the shortened M phase. However, there was no significant

3 reduction in the time between NEBD and anaphase onset (Supplementary Fig. 4C),

4 which indicates that the observed effect does not result from bypassing or

5 premature satisfaction of the spindle assembly checkpoint.

$7 \quad$ LIN-5 recruits dynein to the cell cortex independently of its ability to track the $8 \quad$ MT plus-end

9 If MT plus-end tracking of the dynein complex is not required for spindle

10 positioning, then how does dynein reach the cortex? To find out, we assessed the

11 cortical localization of dynein in both wt and $\Delta e b p$ mutants. Previous studies have

12 attempted to visualize cortical dynein localization by immunohistochemistry ${ }^{11,13}$.

13 While cortical dynein could be observed in two-cell embryos, immunostaining of

14 one-cell embryos did not show clear cortical localization. Time-lapse SDCLM of

15 egfp::dhc-1 one-cell embryos revealed broad and transient regions of DHC-1

16 enrichment at the cortex during anaphase. These were most pronounced during

17 rocking of the spindle. Interestingly, this cortical enrichment of dynein was not

18 perturbed in $\Delta e b p-1 / 3$ or $\Delta e b p-2$ mutant embryos (Fig. 4A). Thus, we conclude that

19 EBP-1/3, and EBP-2-dependent plus-end tracking are not required for the cortical

20 localization of dynein. By contrast, knock-down of lin-5 perturbed cortical dynein

21 localization but did not affect its localization to MT plus-ends (Fig. 4A).

22 Simultaneous dual-color imaging of embryos expressing endogenously-tagged

23 egfp::lin-5 and mcherry::dhc-1 showed that dynein comets do not co-localize with 
1 LIN-5, whereas cortical dynein and LIN-5 co-localize at the cortex (Fig. 4B).

2 Moreover, we previously observed cortical dynein localization in the absence of

3 MTs, following treatment of perm-1(RNAi) embryos with $1 \mu \mathrm{M}$ nocodazole

4 (Portegijs et al., accepted manuscript). In the absence of astral MTs, as confirmed by

5 tubulin and EBP-2 markers, dynein still localized to the cortex, and this localization

6 strongly overlapped with and depended on LIN-5 (Fig. 4C). In addition, we

7 visualized cortical dynein in the early embryo by time-lapse TIRF microscopy.

8 Notably, this revealed two populations of dynein that could be genetically

9 separated at the cortex: a population of bright large spots that represent dynein

10 comets at the cell cortex (Fig. 4D, arrow), and a fraction of smaller, less dynamic

11 spots that represent a LIN-5 dependent population (Fig. 4D, arrowhead). Most

12 notably, the combination of $\Delta e b p-2$ with knock-down of lin-5 resulted in a

13 complete loss of cortical dynein (Fig. 4D, Supplementary Movie 8). Thus, two

14 independent populations of dynein appear present at the cortex: an EBP-2-

15 dependent plus-end tracking and a LIN-5-dependent cortical population. LIN-5 can

16 most likely recruit dynein directly from the cytoplasm to the cortex, which would

17 explain why plus-end tracking of dynein is not required for its cortical localization.

19 Cortical pulling forces remain normal in the absence of end-binding proteins

20 and dynein plus-end tracking.

21 To assess the effect of the absence of EBPs and dynein plus-end tracking on

22 anaphase pulling forces, we imaged embryos by DIC time-lapse microscopy during

23 mitosis. Subsequently, we tracked anterior and posterior pole movements during 
1 anaphase and calculated the average maximum amplitude, which is a read-out for

2 force generation during anaphase ${ }^{55}$. Interestingly, rocking of both the anterior and

3 posterior centrosome was generally not affected by the removal of EBPs. We did

4 find a slight but significant increase in the maximum amplitude for the posterior

5 pole in the $\Delta e b p-1 / 3$ background, but this change was not present in the $\Delta e b p$ -

$6 \quad 1 / 2 / 3$ background (Fig. 5A).

7 To quantify the forces generated during anaphase more directly, we

8 performed spindle severing assays with a focused UV laser beam ${ }^{4}$. Upon severing

9 of the central spindle during anaphase onset, the centrosomes move away from

10 each other with a velocity proportional to the pulling forces acting on their astral

$11 \mathrm{MTs}^{4}$ (Fig. 5B, Supplementary Movie 9). Remarkably, we did not find any significant

12 differences in average peak velocity between any of the $\Delta e b p$ mutants and wild-

13 type embryos for either pole (Fig. 5C). Thus, cortical pulling force generation is

14 robust and remains largely unaltered in the absence of EB proteins. We conclude

15 that dynein plus-end tracking is not required for cortical pulling force generation in

16 the one-cell C. elegans embryo.

17

The EB proteins not only determine dynein plus-end tracking but are also thought to affect several aspects of MT dynamics. The direct effects of EBs on MT dynamics are still unclear, because in vitro and in vivo studies have yielded contradictory results ${ }^{25,56-58}$. The prevailing view is that EBs increase the growth rate as well as the catastrophe frequency of MTs in vitro, but have little effect on the growth rate and suppress catastrophes in cells. However, the effect of complete 
1 loss of EB family members on MT dynamics has not been reported in an established

2 in vivo system such as C. elegans. A hurdle for studying MT dynamics in the one-cell

3 embryo is the extremely dense MT network, with each centrosome of the spindle

4 concurrently nucleating about $300 \mathrm{MTs}^{50}$. This complicates the observation of

5 single MTs by GFP-tubulin labeling, and we could not use +TIP markers as an

6 alternative in this case. Therefore, we imaged the dynamics of labeled tubulin at

7 the cortex with the use of TIRF microscopy. By quantification of the end-on MT-

8 cortex contacts during early anaphase, we found a significant increase in residence

9 time in both $\Delta e b p-2$ and $\Delta e b p-1 / 2 / 3$, but not for $\Delta e b p-1 / 3$, mutants compared to

10 wild type (Fig. 5D, Supplementary Movie 10). This indicates that loss of EBP-2 either

11 reduces MT growth rate, catastrophe frequency or both, thereby possibly allowing

12 prolonged contact with the cell cortex. Interestingly, we also observed a reduced

13 MT density of the spindle midzone in $\Delta e b p-2$ and $\Delta e b p-1 / 2 / 3$ embryos

14 (Supplementary Fig. 5). This led to full or partial bisection of spindles, reminiscent

15 of $s p d-1^{P R C 1}$ knock-down, which diminishes the mechanical strength required for

16 the midzone to counteract the forces acting on the centrosomes ${ }^{59}$. We attribute

17 this effect to a potential reduction in MT polymerization. Collectively, these results

18 indicate that the +TIP network composition, dynein plus-end tracking, and MT

19 dynamics are altered by the loss of the EBPs. However, the net pulling forces are

20 not affected, possibly due to compensation by altered MT dynamics. 


\section{Discussion}

3 In this study, we investigated the recruitment of dynein in the generation of pulling

4 forces that position the mitotic spindle and determine the plane of cell division. We

5 took advantage of the well-established C. elegans one-cell embryo as an in vivo

6 model for spindle positioning, by combining targeted genome editing with high

7 spatial and temporal resolution microscopy, spindle severing assays, RNAi, and

8 drug treatment. Using CRISPR/Cas9-assisted recombineering, we tagged the

9 endogenous dynein complex and created knockout alleles for all three genes

10 encoding plus-end binding proteins of the EB1 family. The ability to study

11 endogenously expressed proteins allowed for reliable phenotypic characterizations

12 and observation of protein dynamics without overexpression.

13 Our first observation was that C-terminal tagging of the dhc- 1 dynein heavy

14 chain causes partial loss of function, as opposed to N-terminal tagging. It is

15 currently unclear whether this translates to dynein in other organisms. N-terminally

16 and C-terminally tagged yeast Dyn 1 were reported to be functional ${ }^{60}$. However,

17 Dyn 1 lacks the C-terminal regulatory extension present in the cytoplasmic dynein

18 heavy chain of other organisms ${ }^{61}$ including $C$. elegans. A BAC transgene with a C-

19 terminally GFP-tagged mouse dynein heavy chain is commonly used in

20 mammalian studies ${ }^{62}$, normally in the presence of the endogenous DHC protein

21 and in cells in culture, which may depend less critically on dynein function. Tagging

22 the N-terminal DHC tail region instead of the C-terminal motor domain might be

23 the best option for future in vivo studies. 
The next interesting observation was the concentration of the dynein

2 complex at the growing MT plus-ends in mitosis. This was previously described for

3 other systems, but to our knowledge not in C. elegans. The detection of dynein

4 plus-end tracking allowed us to quantify whether this transient dynein localization

5 forms part of the mechanism for cortical pulling force generation. Surprisingly,

6 pulling forces and spindle positioning remained unaltered in the absence of dynein

7 plus-end tracking and removal of the entire EB protein family. These observations

8 are in clear contrast with data from budding yeast, which indicate that plus-end

9 tracking provides an active system to deliver dynein to the daughter cell cortex. In vivo and in vitro experiments with S. cerevisiae Dyn1 have implicated the

11 EB1-related protein Bim1 $1^{\mathrm{EB} 1}$, together with Bik1 ${ }^{\mathrm{CLP170}}$, PaC1 ${ }^{\mathrm{LS} 1}$, and Kip2 ${ }^{\mathrm{Kinesin}}$ in

12 dynein plus-end recruitment ${ }^{63,64}$. Yeast dynein is thought to be primed at the MT

13 plus-end for cortical anchorage by relieving its auto-inhibition ${ }^{32,33,65}$. Offloading

14 from the MT plus-end to the cortex is dynactin-dependent, and allows cortical

15 anchorage by Num 1 and correct spindle positioning ${ }^{32,34}$. This two-step mechanism

16 was suggested to locally recruit dynein while at the same time minimizing the

17 need for modulation of motor activity. In yeast, an estimated $30 \%$ of plus-end dynein appears delivered via

19 kinesin-mediated transport, while $70 \%$ is recruited from the cytoplasm ${ }^{34}$. This,

20 together with the finding that Bik $1^{\mathrm{CLP170}}$ can recruit dynein to the MT plus-end in

21 absence of Bim $1^{\mathrm{EB} 166}$, likely explains why depletion of Bim $1^{\mathrm{EB} 1}$ from yeast cells does

22 not completely abolish dynein plus-end tracking ${ }^{63}$. Isolated Dyn1 shows

23 constitutive minus-end-directed processive movement upon dimerization in vitro ${ }^{60}$. 
$1 \quad$ This indicates why kinesin plus-end directed transport is important for keeping

2 Dyn 1 at the plus-end ${ }^{64}$. While we did not explore the role of kinesin motors in the

3 context of dynein plus-end tracking, the effect of EBP-2 depletion suggests that

4 kinesin-mediated transport of dynein does not play a major role in C. elegans.

5 Possibly, C. elegans dynein regulation is more related to the human than to the

6 yeast dynein complex.

In vitro reconstitution studies have shown that the human dynein complex

8 is recruited to MT plus-ends by the large dynactin subunit p150Glued, which binds

9 to growing MT ends through EB1 and CLIP-17037. As a major difference, yeast

10 dynein dimers are active on their own, while mammalian cytoplasmic dynein is in

11 an inactive conformation and requires adaptor-mediated dynactin binding for

12 processive movement ${ }^{67,68}$. If both human and C. elegans dynein are kept inactive at

13 the plus-end, then the 'tug-of-war' with a potential kinesin should not be needed.

14 Kinesin and LIS1 dependent dynein transport has been observed, but its role may

15 be specific for certain cell types such as neurons ${ }^{69}$. However, a recent study

16 indicates that LIS-1 is required for dynein plus-end tracking in C. elegans (Rodriguez

17 Garcia et al., submitted). Interestingly, subcellular location-dependent adaptor

18 proteins bridge dynein and dynactin to activate dynein processivity, including the

19 coiled-coil proteins Bicaudal D at intracellular membranes, Rab11-FIB3 at Rab11-

20 endosomes and Spindly at the kinetochore. It is attractive to hypothesize that LIN-

$215 /$ NuMA acts as a dynein-activating adaptor at the cell cortex.

23 Could MT plus-end tracking of dynein play a role in asymmetric force generation? 
1 In S. cerevisiae, dynein cortical recruitment is polarized by its ability to track the MT

2 plus-end, which directs dynein transport to the bud cortex ${ }^{32}$. However, our data

3 would suggest that dynein plus-end tracking is not in itself a polarizing mechanism

4 in C. elegans. We did not observe any obvious asymmetry in dynein localization to

5 the MT plus-end, and the spindle as well as the distribution of end-on MT contacts

6 were shown to be symmetric in the one-cell embryo ${ }^{50}$. In addition, the presence of

7 cortical dynein and normal spindle positioning in the absence of EBPs indicates

8 that dynein plus-end tracking is not required for asymmetric spindle positioning.

9 This is further supported by data from nocodazole treatment of embryos, which

10 strongly suggests that LIN-5 can recruit dynein directly from the cytoplasm, as

11 opposed to MT-mediated delivery of dynein to the cortex. This is in accordance

12 with observations in nocodazole-treated cultured mammalian cells, which have

13 shown that cortical dynein recruitment does not require MT polymerization. At the

14 same time, the correct distribution of cortical dynein has been reported to depend

15 on a dynamic astral MT network ${ }^{70}$. Thus, in C. elegans and mammalian cells, MT-

16 mediated delivery does not appear necessary for dynein localization to the cortex,

17 but dynamic MTs appear generally required for the correct distribution of dynein at 18 the cortex.

Based on our results, we propose that cortical recruitment of dynein in $C$.

21 elegans occurs by a mechanism different from the off-loading in yeast. We did not

22 observe off-loading or minus-end directed transport of dynein when MT plus-ends

23 reach the cortex. Although rapid diffusion appeared to prevent visualization, the 
1 strong similarity and dependence in localization supports that dynein follows EBP-

22 plus-end association and release. Intriguingly, we found that pulling force

3 generation and spindle positioning are completely normal in the absence of EBPs.

4 This was surprising, as we did observe effects on MT dynamics and +TIP network

5 composition. It is worth noting that a minor dampening of centrosome rocking

6 amplitudes was observed upon perturbation of ebp-2 in a recent study

7 complementary to ours (Rodriguez Garcia et al., submitted). Our quantifications do

8 not reveal such an effect in $\Delta e b p$-2 embryos, which might be explained by the use

9 of different methods of quantification, reporter strains and mutant alleles. dynamics, and what is the relevance of dynein plus-end tracking in the context of

13 spindle positioning? We propose that in a normal situation, dynein plus-end

14 tracking could function as a local enrichment mechanism (Fig. 5E). By

15 concentrating dynein at the plus-end, MTs could efficiently constitute complete

16 FGCs with complexes of Ga-GPR-1/2-LIN-5 waiting at the cortex (Fig. 5E, arrow 1).

17 This local concentration of dynein could also increase the relative amount of complete FGCs present at the cortex. Thus, dynein plus-end tracking could be a

19 back-up mechanism that ensures efficient force generation. In the absence of EBPs

20 and thus dynein plus-end tracking, astral MTs would have to locate cortical

21 complexes that anchor dynein directly from the cytoplasm (Fig. 5E, arrow 2). While

22 this single mechanism was previously hypothesized to be less efficient ${ }^{71}$, we expect

23 that the prolonged cortical residence of MTs might allow for successful probing of 
1 the cortex. In addition, considering that the +TIP is a protein-dense network in

2 which a limited amount of binding sites are available at any given time, cortical

3 FGCs may associate with MTs more efficiently due to decreased crowding of MT

4 tips in EB-depleted cells. Taken together, our work illustrates the complexity and

5 robustness of molecular mechanisms controlling an essential cellular process such

6 as spindle positioning.

7 


\section{$1 \quad$ Materials and Methods}

2

\section{C. elegans strains}

4 A summary of the strains used in this study is included in Table 1. All strains were

5 maintained at $20^{\circ} \mathrm{C}$ as described previously ${ }^{72}$, unless stated otherwise. Worms were

6 grown on plates containing nematode growth medium (NGM) seeded with OP50

7 Escherichia coli bacteria.

\section{Generation of CRISPR/Cas9 repair templates and gRNAs}

10 Homology arms of at least 1500 bp flanking the CRISPR/Cas9 cleavage site were

11 generated by PCR amplification from purified C. elegans genomic DNA using the

12 KOD polymerase (Novagen). PCR products were inserted into the pBSK backbone

13 by Gibson assembly (New England Biolabs). For the generation of ph::egfp::/lov and

14 egfp:::dhc-1, egfp was amplified from pMA-egfp, ph from Pwrt-2::gfp::ph ${ }^{73}$ and lov

15 from gfp::LOVpep::unc-54UTR ${ }^{74}$. For mcherry::dhc-1 and dhc-1::mcherry, codon-

16 optimized mcherry was amplified from TH0563-PAZ-mCherry (a kind gift from A.

17 Hyman). Primers containing overlaps between PCR fragments, linker sequences

18 and mutated gRNA sites were synthesized (Integrated DNA technologies). For the

19 generation of gRNA vectors, oligonucleotides were annealed and inserted into

20 pJJR50 using T4 ligation (New England Biolabs). Vectors were used to transform

21 and purified from DH5alpha competent cells (Qiagen). 


\section{CRISPR/Cas9-mediated genome editing}

2 Injection of adult C. elegans worms in the germ-line was carried out using an

3 inverted microscope micro-injection setup. Injection mixes contained a

4 combination of 30-50 ng/ $\mu$ l Peft-3::cas9 (Addgene ID \#46168 ${ }^{42}, 50-100 \mathrm{ng} / \mu \mathrm{l}$

5 u6::sgRNA with sequences targeted against either cxTi10816, dhc-1, ebp-1, ebp-2 or

$6 e b p-3,30-50 \mathrm{ng} / \mu \mathrm{l}$ of the repair template, $50 \mathrm{ng} / \mu \mathrm{l}$ PAGE-purified $p h a-1$ repair

7 oligonucleotide (Integrated DNA technologies), $60 \mathrm{ng} / \mu \mathrm{l} \mathrm{pJW1285}$ (Addgene ID

$\left.8 \quad \# 61252^{75}\right)$, and $2.5 \mathrm{ng} / \mu \mathrm{l}$ Pmyo-2::tdtomato as a co-injection marker. Animals were

9 grown for $3-5$ days at either 20 or $25^{\circ} \mathrm{C}$ after injection, and transgenic progeny was

10 selected based on either expression of tdTomato in the pharynx or survival at the

11 non-permissive temperature $\left(25^{\circ} \mathrm{C}\right)$. Subsequent assessment of genome editing

12 events was carried out by either visual inspection using a wide-field fluorescence

13 microscope and/or PCR amplification using primers targeting the inserted FP and a

14 genomic region situated outside of the range of homology arms in case of $d h c-1$, or

15 sequences flanking the predicted cut sites as well as an internal control in case of

16 the $\Delta e b p-1 / 2 / 3$ knock-out mutants. The contexts of PCR-confirmed edited genomic

17 loci were further inspected by sequencing (Macrogen Europe).

19 Quantification of embryonic lethality and total brood size

20 In two separate experiments, N2, SV1598, SV1619 and SV1803, or N2, SV1868,

21 SV1872, SV1877 and SV1882 single L4-stage hermaphrodites were placed on OP50

22 feeding plates and kept at $20^{\circ} \mathrm{C}$. Animals were transferred to a new plate every day. 
1 On each plate, embryonic lethality was scored after 24 hours, and brood size 48

2 hours after removal of the parent. Experiments were executed in quadruplicate.

4 Microscopy

5 For time- and stream-lapse imaging embryos were dissected from adult worms on

6 coverslips in $0.8 x$ egg salts buffer $94 \mathrm{mM} \mathrm{NaCl}, 32 \mathrm{mM} \mathrm{KCl}, 2.7 \mathrm{mM} \mathrm{CaCl}$, $2.7 \mathrm{mM}$

$7 \mathrm{MgCl} 2,4 \mathrm{mM}$ HEPES, $\mathrm{pH} 7.5^{76}$, and subsequently mounted on $4 \%$ agarose pads.

8 Live-cell SDCLM imaging of one-cell embryos was performed on a Nikon Eclipse Ti

9 with Perfect Focus System, Yokogawa CSU-X1-A1 spinning disc confocal head, Plan

10 Apo VC 60x N.A. 1.40 oil and S Fluor 100x N.A. 0.5-1.3 (at 1.3, for photo-ablation)

11 objectives, Photometrics Evolve 512 EMCCD camera, DV2 two-channel beam-

12 splitter for simultaneous dual-color imaging, Cobolt Calypso $491 \mathrm{~nm}(100 \mathrm{~mW})$,

13 Cobolt Jive $561 \mathrm{~nm}(100 \mathrm{~mW})$ and Teem Photonics $355 \mathrm{~nm}$ Q-switched pulsed laser

14 controlled with the ILas system (Roper Scientific France/ PICT-IBiSA, Institut Curie,

15 used for photo-ablation), ET-GFP (49002), ET-mCherry (49008) and ET-GFPmCherry

16 (49022) filters, ASI motorized stage MS-2000-XYZ with Piezo Top Plate, and Sutter

17 LB10-3 filter wheel. The microscope was controlled with MetaMorph 7.7 software

18 and situated in a temperature-controlled room at $20^{\circ} \mathrm{C}$. For regular single- and

19 dual-channel imaging experiments, images were acquired in either stream-lapse

20 mode with 100 ms exposure, or time-lapse mode with 500 ms exposure and 5

21 second intervals, unless stated otherwise. Laser power was kept constant within

22 experiments. For spindle bisection assays, spindles were imaged after photo-

23 ablation in stream-lapse mode with 500 ms exposure time. 
1 Simultaneous dual-color TIRF imaging of embryos was performed on a Nikon

2 Eclipse Ti with Perfect Focus System, Nikon Apo TIRF 100x N.A. 1.49 oil objective,

3 Photometrics Evolve 512 EMCCD camera, Optosplit III beam-splitter for

4 simultaneous dual-color imaging, $488 \mathrm{~nm}(150 \mathrm{~mW})$ and Cobolt Jive $561 \mathrm{~nm}(100$

$5 \mathrm{~mW}$ ) lasers, ET-GFP (49002), ET-mCherry (49008) and ET-GFPmCherry (49022) filters,

6 ASI motorized stage MS-2000-XY System for Inversted Microscope Nikon Te/Ti

7 2000, and Sutter LB10-3 filter wheel. Acquisition was controlled with MetaMorph

$8 \quad 7.7$ software and the setup was situated in a temperature-controlled room at $20^{\circ} \mathrm{C}$.

9 For single- and dual-channel imaging experiments, images were acquired in

10 stream-lapse mode with $100 \mathrm{~ms}$ exposure time.

11 Single-color TIRF imaging of embryos was performed on either above-mentioned

12 TIRF setup, or on an identical TIRF setup in which lasers were controlled by the llas-

132 system (Roper Scientific France / PICT-IBiSA, Institut Curie), and image acquisition

14 was controlled with MetaMorph 7.8 software.

15 Live-cell wide-field time-lapse differential interference contrast (DIC) microscopy

16 imaging of embryos was performed on a Zeiss Axioplan upright microscope, with a

17 100x N.A. 1.4 PlanApochroma objective, controlled by AxioVision Rel 4.7 software,

18 at an acquisition rate of 1 image per 2 seconds with constant exposure time and

19 light intensity. Embryos were followed from pronuclear meeting until completion

20 of the first division. Images acquired by SDCLM and TIRF microscopy were

21 prepared for publication in ImageJ by adjusting brightness and contrast,

22 subtracting background and frame averaging as described in figure legends. 


\section{RNA-mediated interference}

2 For RNAi experiments ${ }^{77}$, either the gonads of young adults were injected with

3 double-stranded RNA targeting RNA molecules of interest (perm-1 and perm-1+

4 lin-5) and grown for 20 hours at $15^{\circ} \mathrm{C}$, or L4 animals were grown on RNAi feeding

5 plates for 48 hours at $15^{\circ} \mathrm{C}$ prior to imaging sessions (lin-5, ebp-1/3, ebp-2).

$7 \quad$ UV laser spindle midzone severing

8 Severing of the mitotic spindle was performed as described previously4. AZ224,

9 TH65, SV1874, SV1879 and SV1900 embryos were cultured and imaged at $20^{\circ} \mathrm{C}$,

10 and subjected to spindle bisection at anaphase onset, as judged by GFP::TBB-2 and

11 TBA-2::YFP tubulin localization. Peak centrosome velocities upon spindle severing

12 were subsequently tracked automatically using image intensity thresholding and

13 the FIJI TrackMate plugin.

\section{Data analysis}

16 All intensity profile measurements on SDCLM and TIRF data were generated using

17 ImageJ. Analysis of the timing of key mitotic events and position of the

18 nucleocentrosomal complex and centrosomes from DIC movies of N2, SV1868,

19 SV1872, SV1877 and SV1882 strains was performed by hand using ImageJ.

20 Quantifications of cortical MT residence time were performed by hand using the

21 FIJ plugin MtrackJ. Speeds of EBP-2::GFP and mCherry::DHC-1 comets during

22 metaphase were calculated based on angles of tracks in kymograph data

23 generated in ImageJ with the KymoResliceWide plugin. Numerical data processing 
bioRxiv preprint doi: https://doi.org/10.1101/118935; this version posted March 21, 2017. The copyright holder for this preprint (which was

not certified by peer review) is the author/funder, who has granted bioRxiv a license to display the preprint in perpetuity. It is made available under aCC-BY-NC-ND 4.0 International license.

1 was performed using Excel 2011 (Microsoft). Line and bar graphs were generated

2 using Prism 6 (GraphPad software, inc.).

3

4 


\section{References}

2

3 1. Galli, M. \& Van Den Heuvel, S. Determination of the cleavage plane in early C.

4 elegans embryos. Annu. Rev. Genet. 42, 389-411 (2008).

5 2. Morin, X. \& Bellaïche, Y. Mitotic Spindle Orientation in Asymmetric and

6 Symmetric Cell Divisions during Animal Development. Dev. Cell 21, 102-119

$7 \quad$ (2011).

8 3. Goldstein, B. \& Hird, S. N. Specification of the anteroposterior axis in

9 Caenorhabditis elegans. Development 122, 1467-74 (1996).

10 4. Grill, S. W., Gönczy, P., Stelzer, E. H. \& Hyman, A. A. Polarity controls forces

11 governing asymmetric spindle positioning in the Caenorhabditis elegans

12 embryo. Nature 409, 630-3 (2001).

13 5. Sulston, J. E., Schierenberg, E., White, J. G. \& Thomson, J. N. The embryonic

14 cell lineage of the nematode Caenorhabditis elegans. Dev. Biol. 100, 64-119

15 (1983).

16 6. Lorson, M. A., Horvitz, H. R. \& Van Den Heuvel, S. LIN-5 is a novel component 17 of the spindle apparatus required for chromosome segregation and

18 cleavage plane specification in Caenorhabditis elegans. J. Cell Biol. 148, 73-

$1986(2000)$.

20 7. Gotta, M. \& Ahringer, J. Distinct roles for Galpha and Gbetagamma in

21 regulating spindle position and orientation in Caenorhabditis elegans

22 embryos. Nat. Cell Biol. 3, 297-300 (2001).

23 8. Srinivasan, D. G., Fisk, R. M., Xu, H. \& Van Den Heuvel, S. A complex of LIN-5 
and GPR proteins regulates $G$ protein signaling and spindle function in $C$

2 elegans. Genes Dev. 17, 1225-1239 (2003).

3 9. Gotta, M., Dong, Y., Peterson, Y. K., Lanier, S. M. \& Ahringer, J. Asymmetrically Distributed C . elegans Homologs of AGS3 / PINS Control Spindle Position in the Early Embryo. Curr. Biol. 13, 1029-1037 (2003).

10. Colombo, K. et al. Translation of polarity cues into asymmetric spindle positioning in Caenorhabditis elegans embryos. Science. 300, 1957-1961 (2003).

11. Nguyen-Ngoc, T., Afshar, K. \& Gönczy, P. Coupling of cortical dynein and G alpha proteins mediates spindle positioning in Caenorhabditis elegans. Nat. Cell Biol. 9, 1294-302 (2007).

12. Kardon, J. R. \& Vale, R. D. Regulators of the cytoplasmic dynein motor. Nat. Rev. Mol. Cell Biol. 10, 854-65 (2009).

13. Kotak, S., Busso, C. \& Gönczy, P. Cortical dynein is critical for proper spindle positioning in human cells. J. Cell Biol. 199, 97-110 (2012).

14. Gusnowski, E. M. \& Srayko, M. Visualization of dynein-dependent microtubule gliding at the cell cortex: implications for spindle positioning. J. Cell Biol. 194, 377-86 (2011).

15. Hendricks, A. G. et al. Dynein tethers and stabilizes dynamic microtubule plus ends. Curr. Biol. 22, 632-637 (2012).

16. Laan, L. et al. Cortical dynein controls microtubule dynamics to generate pulling forces that position microtubule asters. Cell 148, 502-14 (2012).

17. Pearson, C. G. \& Bloom, K. Dynamic microtubules lead the way for spindle 
1 positioning. Nat. Rev. Mol. Cell Biol. 5, 481-492 (2004).

2 18. Akhmanova, A. \& Steinmetz, M. O. Control of microtubule organization and dynamics: two ends in the limelight. Nat. Rev. Mol. Cell Biol. 16, 711-26 (2015).

19. Honnappa, S. et al. Key Interaction Modes of Dynamic +TIP Networks. Mol. Cell 23, 663-671 (2006).

20. Zimniak, T., Stengl, K., Mechtler, K. \& Westermann, S. Phosphoregulation of the budding yeast EB1 homologue Bim1p by Aurora/Ipl1 p. J. Cell Biol. 186, 379-391 (2009).

22. Tamura, N. et al. A proteomic study of mitotic phase-specific interactors of EB1 reveals a role for SXIP-mediated protein interactions in anaphase onset. Biol. Open 4, 155-69 (2015).

23. Bieling, P. et al. Reconstitution of a microtubule plus-end tracking system in vitro. Nature 450, 1100-1105 (2007).

24. Maurer, S. P., Fourniol, F. J., Bohner, G., Moores, C. A. \& Surrey, T. EBs

21 25. Zhang, R., Alushin, G. M., Brown, A. \& Nogales, E. Mechanistic origin of 849-859 (2015). 
1 26. Vaughan, K. T., Hughes, S. H., Echeverri, C. J., Faulkner, N. F. \& Vallee, R. B. Colocalization of dynactin and cytoplasmic dynein with CLIP-170 at microtubule distal ends. J. Cell Sci. 112, 1437-1447 (1999).

27. Han, G. et al. The aspergillus cytoplasmic dynein heavy chain and nudf localize to microtubule ends and affect microtubule dynamics. Curr. Biol. 11, 719-724 (2001).

28. Lenz, J. H., Schuchardt, I., Straube, a \& Steinberg, G. A dynein loading zone for retrograde endosome motility at microtubule plus-ends. $E M B O$ J. 25, $2275-2286$ (2006).

29. Kobayashi, T. \& Murayama, T. Cell cycle-dependent microtubule-based dynamic transport of cytoplasmic dynein in mammalian cells. PLoS One 4, (2009).

30. Kirschner, M. \& Mitchison, T. Beyond self-assembly: From microtubules to morphogenesis. Cell 45, 329-342 (1986).

31. Vaughan, P. S., Miura, P., Henderson, M., Byrne, B. \& Vaughan, K. T. A role for regulated binding of $\mathrm{p} 150$ (Glued) to microtubule plus ends in organelle transport. J. Cell Biol. 158, 305-319 (2002).

32. Sheeman, B. et al. Determinants of S. cerevisiae dynein localization and activation: Implications for the mechanism of spindle positioning. Curr. Biol. $13,364-372$ (2003).

33. Lee, W. L., Oberle, J. R. \& Cooper, J. A. The role of the lissencephaly protein Pac1 during nuclear migration in budding yeast. J. Cell Biol. 160, 355-364 (2003). 
1 34. Markus, S. M. \& Lee, W. L. Regulated offloading of cytoplasmic dynein from microtubule plus ends to the cortex. Dev. Cell 20, 639-651 (2011).

35. Lansbergen, G. et al. Conformational changes in CLIP-170 regulate its binding to microtubules and dynactin localization. J. Cell Biol. 166, 1003$1014(2004)$.

36. Berrueta, L., Tirnauer, J. S., Schuyler, S. C., Pellman, D. \& Bierer, B. E. The APCassociated protein EB1 associates with components of the dynactin complex and cytoplasmic dynein intermediate chain. Curr. Biol. 9, 425-428 (1999).

37. Duellberg, C. et al. Reconstitution of a hierarchical +TIP interaction network controlling microtubule end tracking of dynein. Nat. Cell Biol. 16, (2014).

38. Waaijers, S. et al. CRISPR/Cas9-targeted mutagenesis in Caenorhabditis elegans. Genetics 195, 1187-91 (2013).

39. Cho, S. W., Kim, S., Kim, J. M. \& Kim, J.-S. Targeted genome engineering in human cells with the Cas9 RNA-guided endonuclease. Nat. Biotechnol. 31, 230-2 (2013).

40. Mali, P. et al. RNA-guided human genome engineering via Cas9. Science 339, 823-6 (2013).

41. Ran, F. A. et al. Double nicking by RNA-guided CRISPR Cas9 for enhanced genome editing specificity. Cell 154, 1380-9 (2013).

42. Friedland, A. E. et al. Heritable genome editing in C. elegans via a CRISPRCas9 system. Nat. Methods 10, 37-54 (2015).

43. Gassmann, R. et al. A new mechanism controlling kinetochore-microtubule interactions revealed by comparison of two dynein-targeting components: 
SPDL-1 and the Rod/Zwilch/Zw10 complex. Genes Dev. 22, 2385-99 (2008).

2 44. Hutchins, J. R. a et al. Systematic analysis of human protein complexes identifies chromosome segregation proteins. Science 328, 593-9 (2010).

45. Ellefson, M. L. \& McNally, F. J. CDK-1 inhibits meiotic spindle shortening and dynein-dependent spindle rotation in C. elegans. J. Cell Biol. 193, 1229-1244 (2011). cytoplasmic dynein heavy chain in Caenorhabditis elegans with fast-acting temperature-sensitive mutations. Mol. Biol. Cell 16, 1200-1212 (2005).

47. Kimura, K. \& Kimura, A. Intracellular organelles mediate cytoplasmic pulling

48. Praitis, V., Casey, E., Collar, D. \& Austin, J. Creation of low-copy integrated force for centrosome centration in the Caenorhabditis elegans early embryo.

51. Skop, A. R. \& White, J. G. The dynactin complex is required for cleavage plane specification in early Caenorhabditis elegans embryos. Curr. Biol. 8, 1110-

23 52. Redemann, S. et al. Membrane invaginations reveal cortical sites that pull on 
1 mitotic spindles in one-cell C. elegans embryos. PLoS One 5, e12301 (2010).

2 53. Frøkjær-Jensen, C. et al. Single copy insertion of transgenes in C. elegans. Nat. Genet. 40, 1375-1383 (2008).

54. Slep, K. C. \& Vale, R. D. Structural Basis of Microtubule Plus End Tracking by XMAP215, CLIP-170, and EB1. Mol. Cell 27, 976-991 (2007).

55. Pecreaux, J. et al. Spindle oscillations during asymmetric cell division require a threshold number of active cortical force generators. Curr. Biol. 16, $2111-$

56. Komarova, Y. et al. Mammalian end binding proteins control persistent microtubule growth. J. Cell Biol. 184, 691-706 (2009).

57. Zanic, M., Widlund, P. O., Hyman, A. a \& Howard, J. Synergy between

58. Maurer, S. P. et al. EB1 accelerates two conformational transitions important for microtubule maturation and dynamics. Curr. Biol. 24, 372-384 (2014).

59. Verbrugghe, K. J. C. \& White, J. G. SPD-1 is required for the formation of the

60. Reck-Peterson, S. L. et al. Single-Molecule Analysis of Dynein Processivity and Stepping Behavior. Cell 126, 335-348 (2006).

21 61. Nicholas, M. P. et al. Control of cytoplasmic dynein force production and processivity by its C-terminal domain. Nat. Commun. 6, 1-8 (2015).

23 62. Poser, l. et al. BAC TransgeneOmics: a high-throughput method for 
exploration of protein function in mammals. Nat. Methods 5, 409-15 (2008).

2 63. Carvalho, P., Gupta, M. L., Hoyt, M. A. \& Pellman, D. Cell cycle control of kinesin-mediated transport of Bik1 (CLIP-170) regulates microtubule stability and dynein activation. Dev. Cell 6, 815-829 (2004).

64. Roberts, A. J., Goodman, B. S. \& Reck-Peterson, S. L. Reconstitution of dynein transport to the microtubule plus end by kinesin. Elife 3, e02641 (2014).

65. Steven M. Markus, S. M., Punch, J. J. \& Lee, W.-L. Motor and Tail Dependent Targeting of Dynein to Microtubule Plus-Ends and the Cell Cortex. Curr. Biol.

4, 196-205 (2009).

66. Caudron, F., Andrieux, A., Job, D. \& Boscheron, C. A new role for kinesindirected transport of Bik1p (CLIP-170) in Saccharomyces cerevisiae. J. Cell Sci. $121,1506-13$ (2008).

67. McKenney, R. J., Huynh, W., Tanenbaum, M. E., Bhabha, G. \& Vale, R. D. Activation of cytoplasmic dynein motility by dynactin-cargo adapter complexes. Science. 345, 337-341 (2014).

68. Schlager, M. A. et al. Bicaudal D Family Adaptor Proteins Control the Velocity of Dynein-Based Movements. Cell Rep. 8, 1248-1256 (2014). of cytoplasmic dynein. EMBO J. 27, 2471-2483 (2008).

70. Tame, M. A. et al. Astral microtubules control redistribution of dynein at the cell cortex to facilitate spindle positioning. Cell Cycle 13, 1162-1170 (2014).

22 71. Ananthanarayanan, V. et al. Dynein motion switches from diffusive to directed upon cortical anchoring. Cell 153, (2013). 
1 72. Brenner, S. The genetics of Caenorhabditis elegans. Genetics 77, 71-94

2 (1974).

73. Wildwater, M., Sander, N., de Vreede, G. \& van den Heuvel, S. Cell shape and Wnt signaling redundantly control the division axis of $C$. elegans epithelial stem cells. Development 138, 4375-4385 (2011).

74. Harterink, M. et al. Light-controlled intracellular transport in Caenorhabditis elegans. Curr. Biol. 26, R153-R154 (2016).

75. Ward, J. D. Rapid and precise engineering of the Caenorhabditis elegans genome with lethal mutation co-conversion and inactivation of NHEJ repair. Genetics 199, 363-77 (2015).

76. Tagawa, a, Rappleye, C. a \& Aroian, R. V. Pod-2, along with pod-1, defines a new class of genes required for polarity in the early Caenorhabditis elegans embryo. Dev. Biol. 233, 412-24 (2001).

77. Fire, A. et al. Potent and specific genetic interference by double-stranded RNA in Caenorhabditis elegans. Nature 391, 806-811 (1998). 


\section{Acknowledgements}

2

3 We thank all of the members of the van den Heuvel, Akhmanova and Boxem

4 groups for helpful discussions and general support. We acknowledge Wormbase

5 and the Biology Imaging Center at the Faculty of Sciences, Department of Biology,

6 Utrecht University, and especially llya Grigoriev and Eugene Katrukha for support

7 regarding the technical aspects of microscopy and data analysis. Some strains were

8 provided by the Caenorhabditis Genetics Center (CGC), which is funded by NIH

9 Office of Research Infrastructure Programs (P40 OD010440). This work was

10 supported by a European Research Council (ERC) Synergy grant 609822 to A.A., and

11 is part of program CW711.011.01 (SvdH) financed by the Netherlands

12 Organization for Scientific Research (NWO). 


\section{Author contributions}

2

3 AA, RS and SvdH designed the study, analyzed the data and wrote the paper. RS

4 performed the experiments.

5

6

7 Competing financial interests statement

8

9 The authors declare no competing financial interests. 
bioRxiv preprint doi: https://doi.org/10.1101/118935; this version posted March 21, 2017. The copyright holder for this preprint (which was

not certified by peer review) is the author/funder, who has granted bioRxiv a license to display the preprint in perpetuity. It is made available under aCC-BY-NC-ND 4.0 International license.

\begin{tabular}{|c|c|}
\hline I. Table 1 & \\
\hline Strain & Genotype \\
\hline N2 & Wild type \\
\hline SV1598 & he248[dhc-1::mcherry] \\
\hline SV1619 & he250[mcherry::dhc-1] \\
\hline SV1635 & he244 [egfp::lin-5]; he250[mcherry::dhc-1] \\
\hline TH65 & ddls15 [[unc-119(+) + C47B2.3(genomic)::YFP]] \\
\hline AZ224 & Ruls57[Ppie-1::gfp::tbb-2] \\
\hline SV1702 & Ruls57[Ppie-1::gfp::tbb-2]; he250[mcherry::dhc-1] \\
\hline SV1703 & he258[Peft-3::ph::egfp::Iov::tbb-2-3'UTR]; he250[mcherry::dhc-1] \\
\hline SV1803 & he264[egfp:::dhc-1] \\
\hline SV1840 & ojls5[pie-1::GFP::dnc-1 + unc-119(+)]; he250[mcherry::dhc-1] \\
\hline SV1841 & ojls57[Ppie-1::gfp::dnc-2 unc-119(+)]; he250[mcherry::dhc-1] \\
\hline SV1857 & abcls3[Ppie-1::ebp-2::gfp; unc-119(+)]; he250[mcherry::dhc-1] \\
\hline SV1868 & he278[ $\Delta$ ebp-2] \\
\hline SV1872 & he279[ $\Delta$ ebp-1, $\Delta$ Y59A8B.25, $\Delta$ ebp-3] \\
\hline SV1873 & he278[ $\Delta$ ebp-2]; he263[egfp:::dhc-1] \\
\hline SV1874 & he278[ $\Delta$ ebp-2]; Ruls57[Ppie-1::gfp::tbb-2] \\
\hline SV1877 & he278[ $\Delta$ ebp-2]; he279[ $\Delta$ ebp-1, $\Delta$ Y59A8B.25, $\Delta$ ebp-3] \\
\hline SV1878 & he279[ $\Delta$ ebp-1, $\Delta$ Y59A8B.25, $\Delta$ ebp-3]; he263[egfp::dhc-1] \\
\hline SV1879 & he279[ $\Delta$ ebp-1, $\Delta$ Y59A8B.25, $\Delta$ ebp-3]; ddls15 [[unc-119(+) + C47B2.3(genomic)::YFP]] \\
\hline SV1900 & [unc-119(+) + C47B2.3(genomic)::YFP]; he278[ $\Delta$ ebp-2]; he279[ $\Delta$ ebp-1, $\Delta$ Y59A8B.25, $\Delta$ ebp-3] \\
\hline
\end{tabular}




\section{Figures}

3 Figure 1. Endogenous tagging of dhc-1 using CRISPR/Cas9.

4 (A) Schematic representation of $d h c-1$ tagging strategies. Blue boxes represent the exons of the $d h c-1$ locus on chromosome I. Red and green boxes represent mcherry

6 and egfp sequences respectively. Orange boxes indicate linkers. Dynein cartoons

7 (right) indicate the location of FPs in DHC-1 for C- (upper) and N-terminal (lower)

8 strategies. (B) Quantification of embryonic lethality (left Y-axis) and brood size

9 (right $\mathrm{Y}$-axis) of $d$ hc- 1 strains. Bars represent average $(\mathrm{N}=4$ replicates $)+\mathrm{SD},{ }^{* * * *} \mathrm{P}<$

100.0001 compared to wt. No indication means no significant difference to wt.

11 Unpaired Welch Student's t-test. (C) Wide-field fluorescence microscopy image of

12 adult animal expressing mCherry::DHC-1. Blow-up indicates expression of

13 mCherry::DHC-1 in the germ-line. Scale bar, $50 \mu \mathrm{m}$. (D) Representative spinning

14 disk confocal microscopy images of single eGFP::DHC-1 embryo in prophase (left),

15 metaphase (middle) and anaphase (right). Scale bar, $5 \mu \mathrm{m}$. All images were taken

16 with the same exposure time and laser power, anterior to the left. 
A

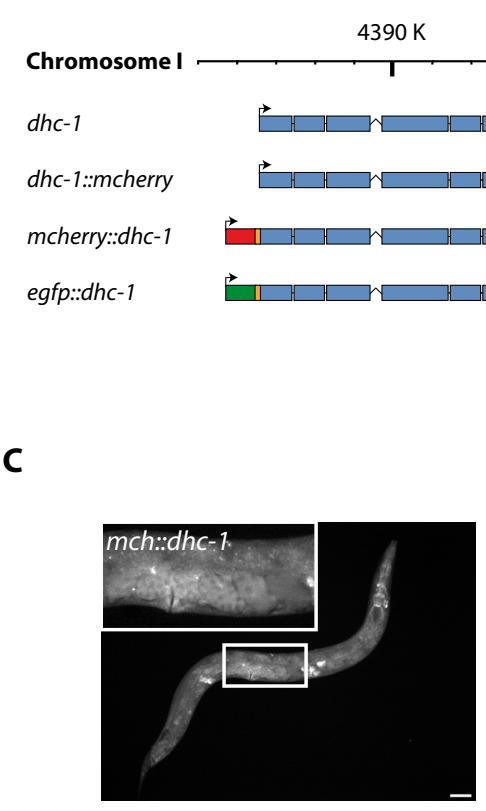

B

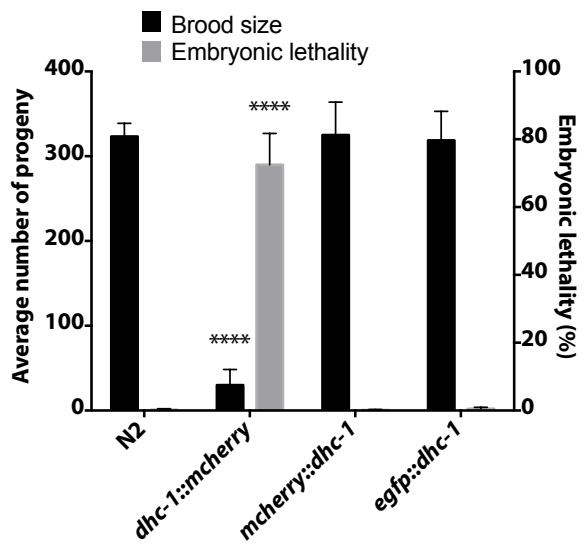

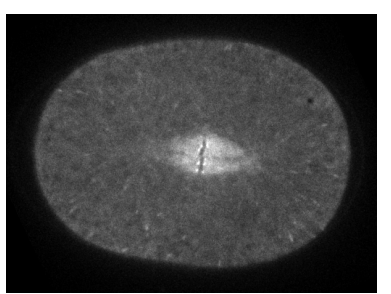

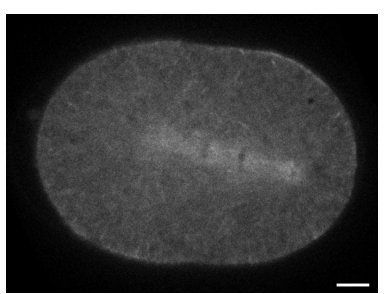

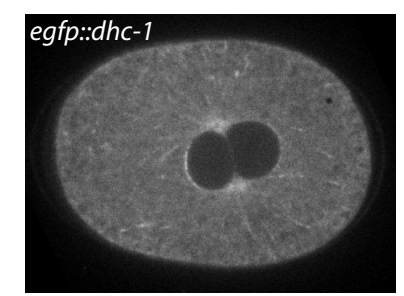

\section{D}




\section{Figure 2. Dynein tracks MT plus-ends during mitosis.}

2 (A, B) Representative simultaneous dual-color SDCLM images of one-cell embryos

3 during mitosis. mCherry::DHC-1 (red), GFP::TBB-2 (MTs, green, A), EBP-2::GFP (plus-

4 end, green, B). Arrow indicates dynein plus-end tracking. Yellow box indicates the

5 blow-up depicted in (C). (C) Intensity profile analysis of mCherry::DHC-1 (red) and

6 EBP-2::GFP (green) comets. Length in pixels, intensity in average ( $\mathrm{N}=10$ comets)

7 percentage of maximum. (D) Quantification of mCherry::DHC-1 (red, $\mathrm{N}=57$ comets)

8 and EBP-2::GFP (green, $\mathrm{N}=116$ comets) average comet velocity $(\mu \mathrm{m} / \mathrm{s})$ during

9 metaphase. Bars represent average + SD, ns not significant. $(E, F)$ Representative

10 simultaneous dual-color TIRF images of mCherry::DHC-1 and GFP::TBB-2 (tubulin)

11 (E) or EBP-2::GFP (F) localization during early anaphase. Graphs indicate intensity

12 profile of mCherry::DHC-1 (red) and GFP::TBB-2 (E) or EBP-2::GFP (F) decoration of

13 the MT plus-end. Length in pixels, intensity in average $(N=10$ comets $E, F)$

14 percentage of maximum. (G) Representative simultaneous dual-color SDCLM

15 images of one-cell embryo expressing mCherry::DHC-1 (red) and PH::eGFP (green,

16 plasma membrane). Panels 4-6 are kymographs of the membrane invagination

17 indicated with the arrow in panel 3. Graph indicates single intensity profile analysis

18 of mCherry::DHC-1 (red) and PH::eGFP as measured along the box delineated in

19 kymograph, representative of $\mathrm{N}=25$ events. Images are averages of 5 consecutive

20 frames taken from 100 ms stream-lapse movies, after background subtraction by a 21 gaussian blur filter. Scale bars, $1 \mu \mathrm{m}$. 
bioRxiv preprint doi: https://doi.org/10.1101/118935; this version posted March 21, 2017. The copyright holder for this preprint (which was

not certified by peer review) is the author/funder, who has granted bioRxiv a license to display the preprint in perpetuity. It is made

A

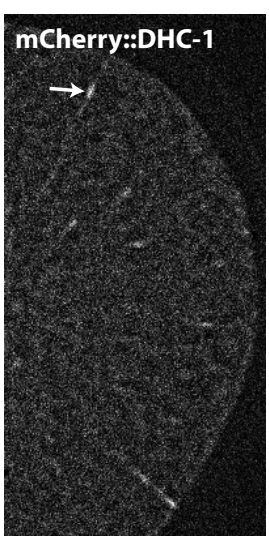

C

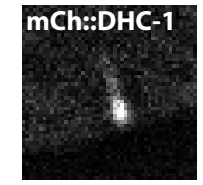

EBP-2:GFP
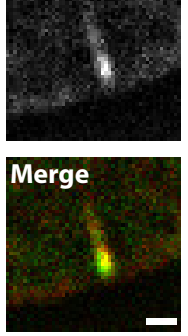

E
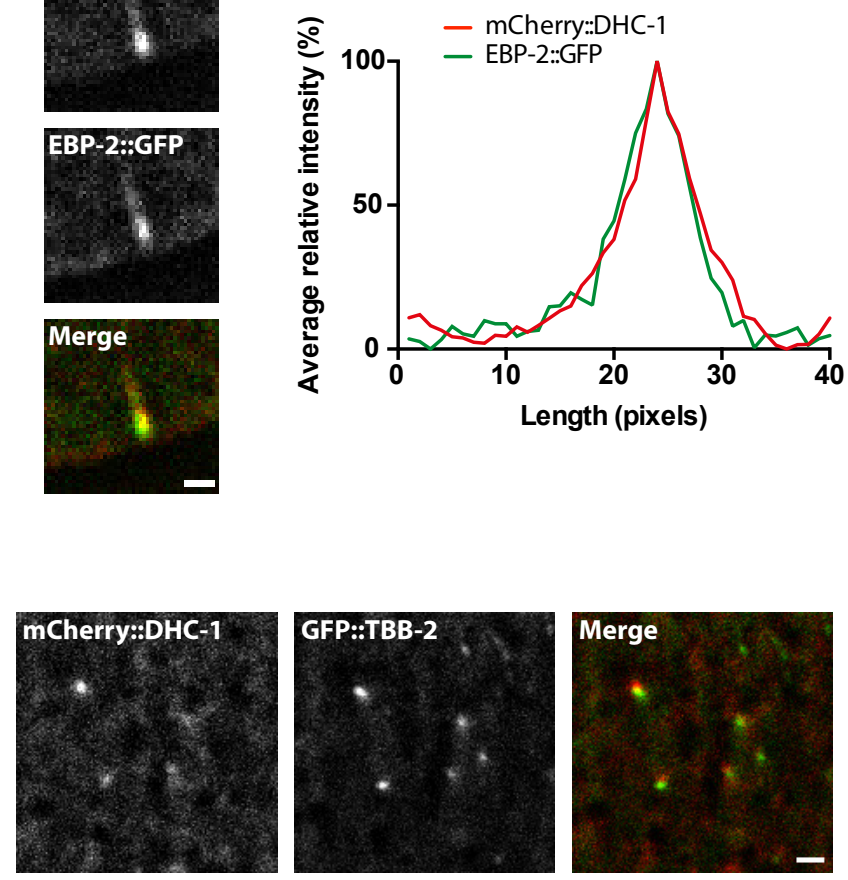

$\mathbf{F}$
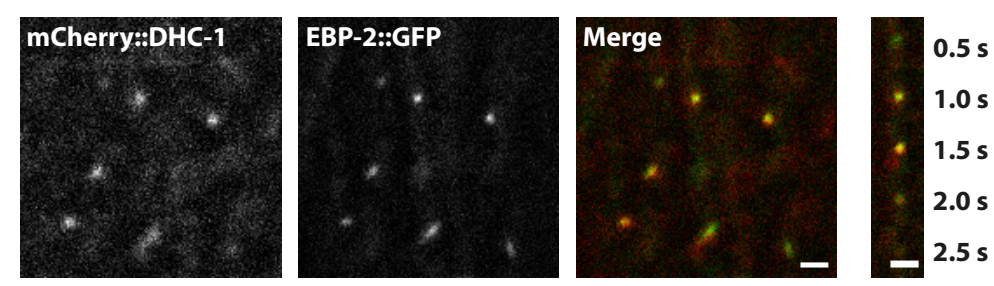
available under aCC-BY-NC-ND
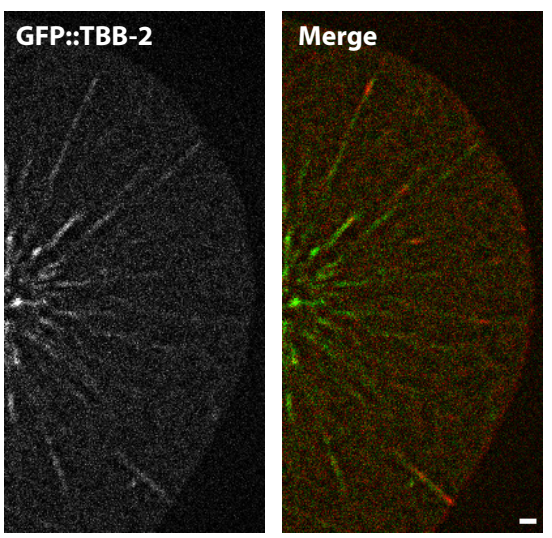

\section{D}

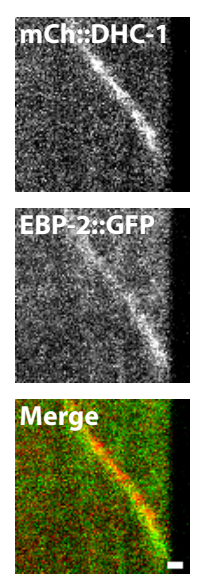

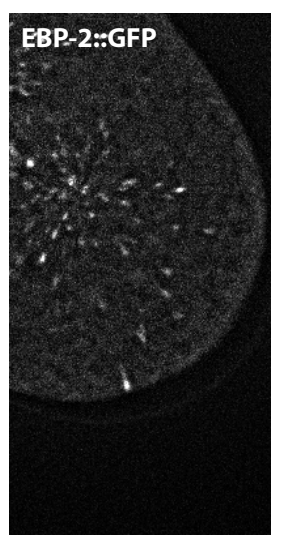

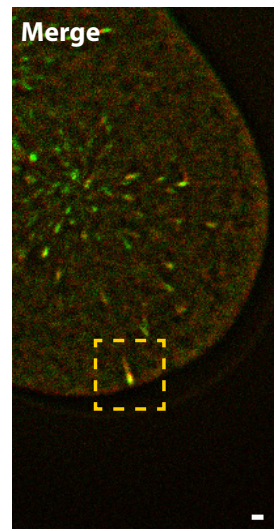

- mCherry::DHC-1

- EBP-2::GFP

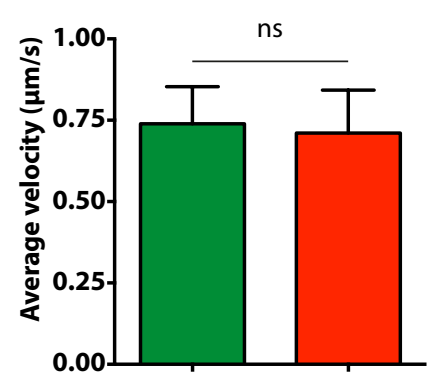

G
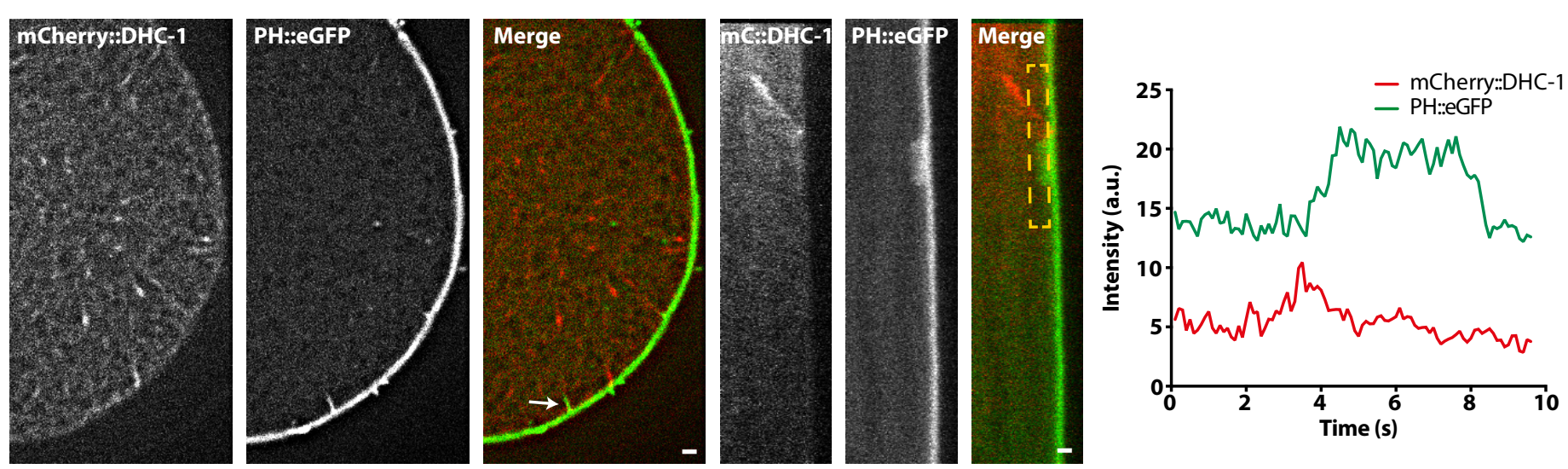


\section{Figure 3. Analysis of dynein plus-end tracking and development in $\Delta e b p$}

\section{2 knock-outs.}

3 (A) Representative SDCLM images of one-cell embryos expressing eGFP::DHC-1

4 during metaphase, either untreated (wt, left) or following RNAi of indicated ebp

5 gene(s) (middle, right). Blow-ups indicate the presence or absence of MT plus-end

6 tracking, which was confirmed in $\mathrm{N}=5$ embryos per condition. Images are averages

7 of 5 consecutive frames taken from 100 ms stream-lapse movies, after background

8 subtraction by a gaussian blur filter. (B) Schematic representation of $e b p-1 / 3$ and

$9 e b p-2$ endogenous knock-out strategies. Blue and green boxes represent relevant

10 genes mentioned in the text. Red boxes indicate the genetic duplication on

11 chromosome $\mathrm{V}$ mentioned in the text. Grey bars represent the $\Delta e b p-1 / 3$ and $\Delta e b p-2$

12 deletions. (C) Representative SDCLM images of one-cell embryos expressing

13 eGFP::DHC-1 during mitosis, in a wt (left), $\Delta e b p-1 / 3$ (middle) or $\Delta e b p-2$ (right)

14 genetic background. Blow-ups indicate the presence or absence of MT plus-end

15 tracking, which was confirmed in $\mathrm{N}=5$ embryos per condition. Images are selected

16 frames from 1000 ms exposure time-lapse movies, processed by background

17 correction. (D) Quantification of embryonic lethality and brood size of $\Delta e b p$

18 mutants. Bars represent average $(\mathrm{N}=4$ replicates $)+\mathrm{SD},{ }^{*} \mathrm{P}<0.05,{ }^{* *} \mathrm{P}<0.01$

19 compared to wt. Unpaired Welch Student's t-test. (E) Representative single images

20 taken from time-lapse DIC movies of $\mathrm{N} 2, \Delta e b p-2, \Delta e b p-1 / 3$ and $\Delta e b p-1 / 2 / 3$ one-cell

21 embryos, aligned in time with PNM (first), centration (second), PSD (third) and

22 cytokinesis completion (fourth column) in wild-type (N2). Times relative to

23 pronuclear meeting are indicated in seconds for every frame. Scale bars, $5 \mu \mathrm{m}$. 
Fig. 3 bioRxiv preprint doi: https://doi.org/10.1101/118935; this version posted March 21, 2017. The copyright holder for this preprint (which was not certified by peer review) is the author/funder, who has granted bioRxiv a license to display the preprint in perpetuity. It is made available under aCC-BY-NC-ND 4.0 International license.

A
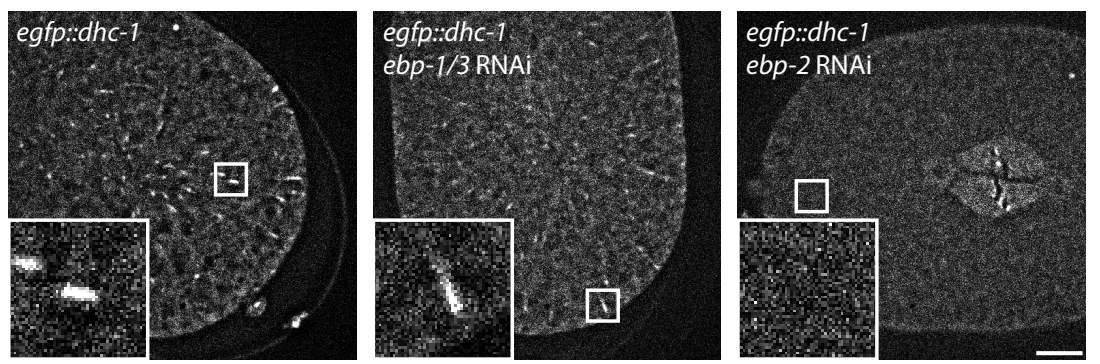

B
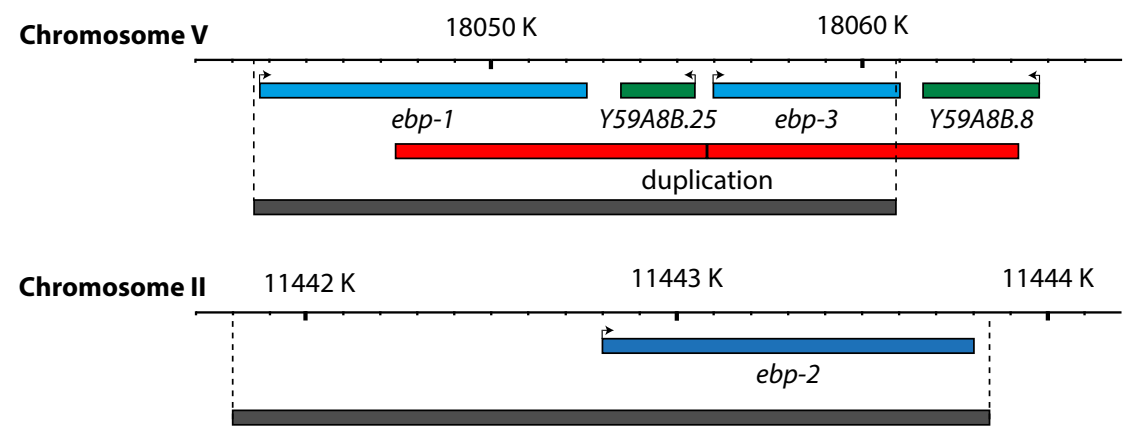

C
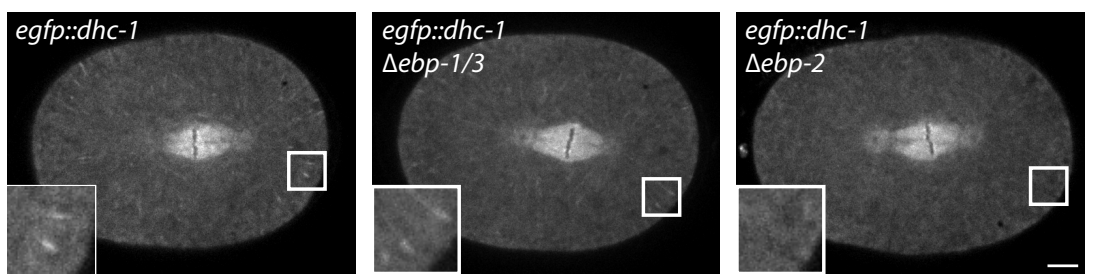

D

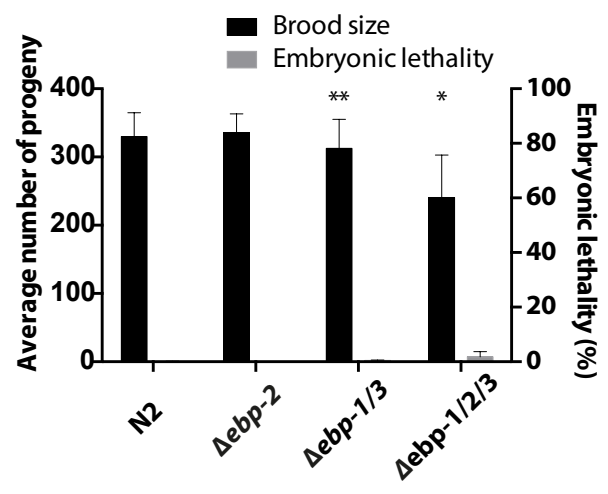

E
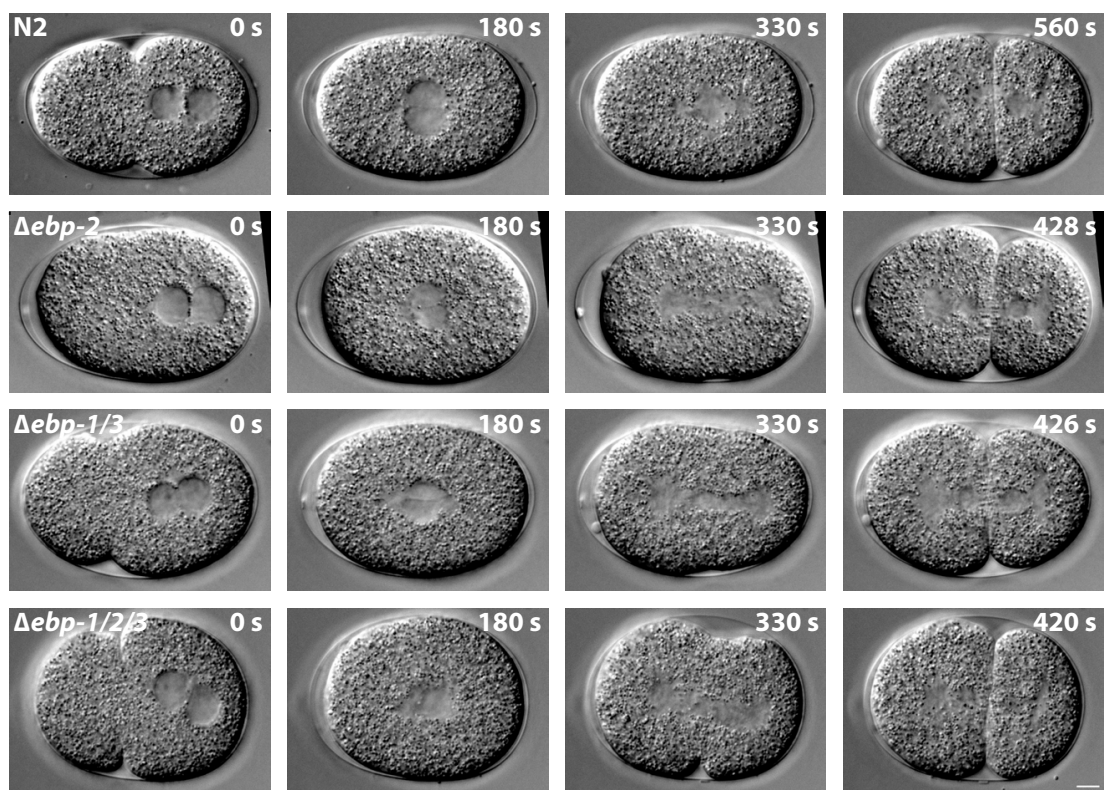
1 Figure 4. Cortical localization of dynein depends on LIN-5, but not on EBP-2mediated plus-end tracking.

3 (A) Representative SDCLM images of eGFP::DHC-1 localization during anaphase,

4 under different RNAi conditions and in various genetic backgrounds as indicated

5 for each panel. Graphs indicate intensity profiles of eGFP::DHC-1 as measured in

6 boxes indicated in each superimposed panel. Length in pixels, intensity relative to

7 cytoplasmic values in average ( $\mathrm{N}=24$ measurements, 4 embryos per condition)

8 percentage of maximum. Images are selected frames from $1000 \mathrm{~ms}$ exposure time-

9 lapse movies, processed with background correction. Scale bar, $5 \mu \mathrm{m}$. (B)

10 Representative simultaneous dual-color SDCLM images of mCherry::DHC-1 (red)

11 and eGFP::LIN-5 (green) localization during mitosis. Arrow indicates dynein plus-

12 end tracking, arrowhead indicates co-localization of dynein and LIN-5 at the cortex,

13 as confirmed in $\mathrm{N}=5$ embryos. Images are averages of 10 consecutive frames taken

14 from 100 ms stream-lapse movie, after background subtraction by a gaussian blur

15 filter. Scale bar, $1 \mu \mathrm{m}$. (C) Representative SDCLM images of either eGFP::DHC-1 or

16 mCherry::DHC-1; eGFP::LIN-5, in embryos treated with perm-1 or perm-1 + lin-5

$17 \mathrm{RNAi}$, and with $1 \mu \mathrm{m}$ nocodazole. Graphs indicate intensity profiles of eGFP::DHC-1

18 or mCherry::DHC-1 (red) and eGFP::LIN-5 (green) as measured in boxes delineated

19 in each superimposed panel. Length in pixels, intensity relative to cytoplasmic

20 values in average ( $\mathrm{N}=10$ measurements, 10 embryos per condition) percentage of

21 maximum. (D) Representative TIRF images of cortical eGFP::DHC-1 during early

22 anaphase, under different RNAi conditions and in various genetic backgrounds as

23 indicated for each panel. Arrow indicates large dot (plus-end dynein), arrowhead 
1 indicates small dot (cortical dynein), as discussed in the text and observed for $\mathrm{N}=6$

2 embryos per condition. Images are averages of 10 consecutive frames taken from

350 ms stream-lapse movie, after background subtraction by a gaussian blur filter.

4 Scale bar, $1 \mu \mathrm{m}$. Scale bar, $5 \mu \mathrm{m}$.

5 
Fig. 4 bioRxiv preprint doi: https://doi.org/10.1101/118935; this version posted March 21, 2017. The copyright holder for this preprint (which was not certified by peer review) is the author/funder, who has granted bioRxiv a license to display the preprint in perpetuity. It is made

A
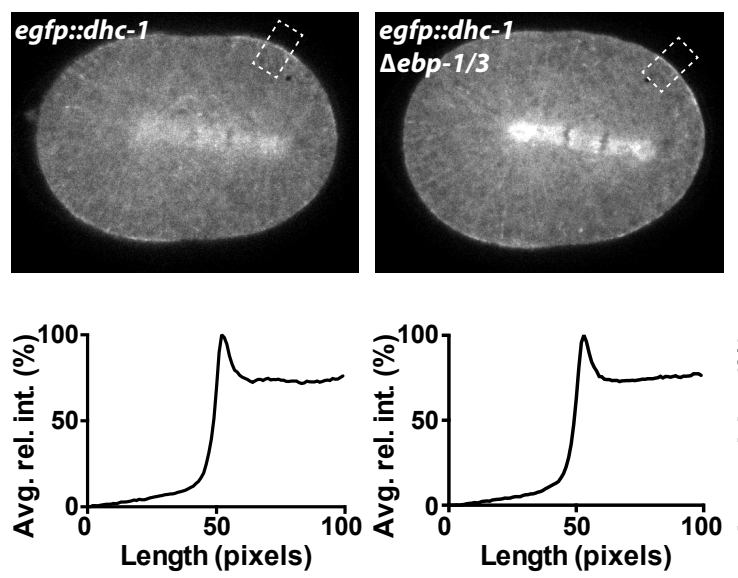

B
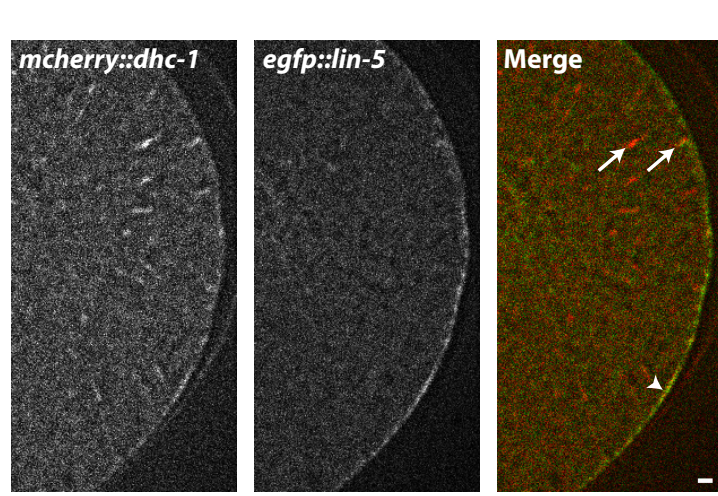

C
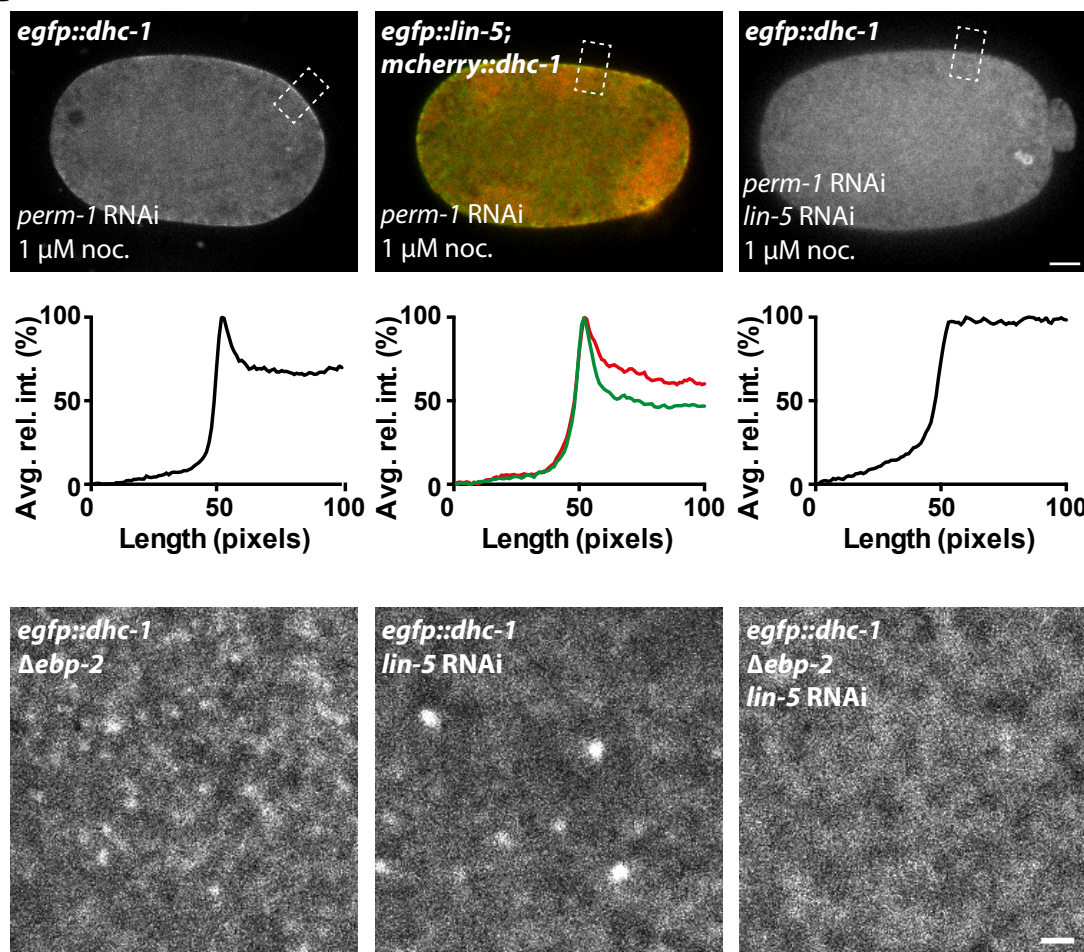
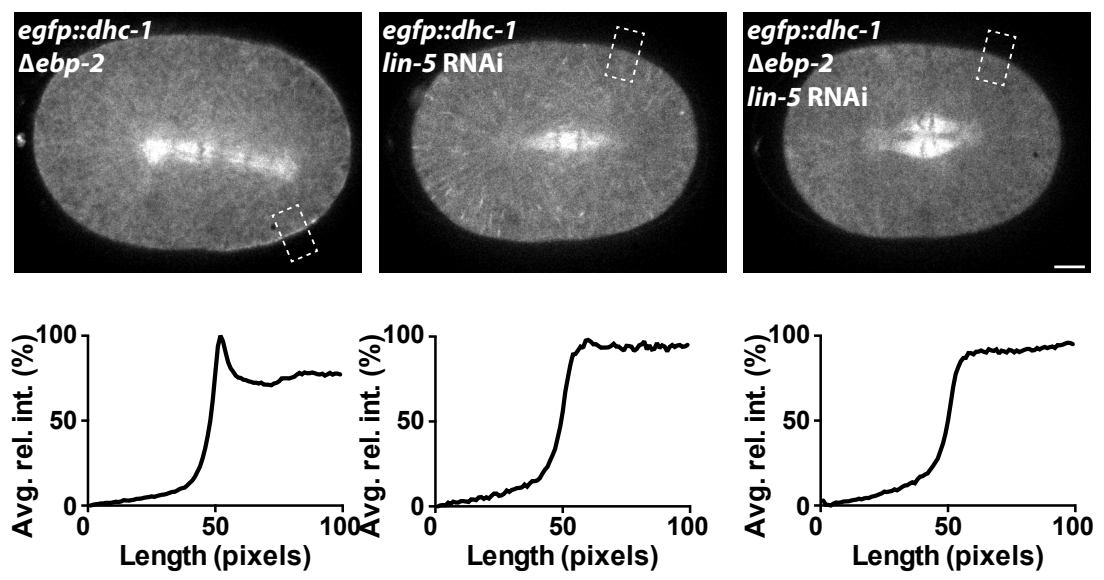

D
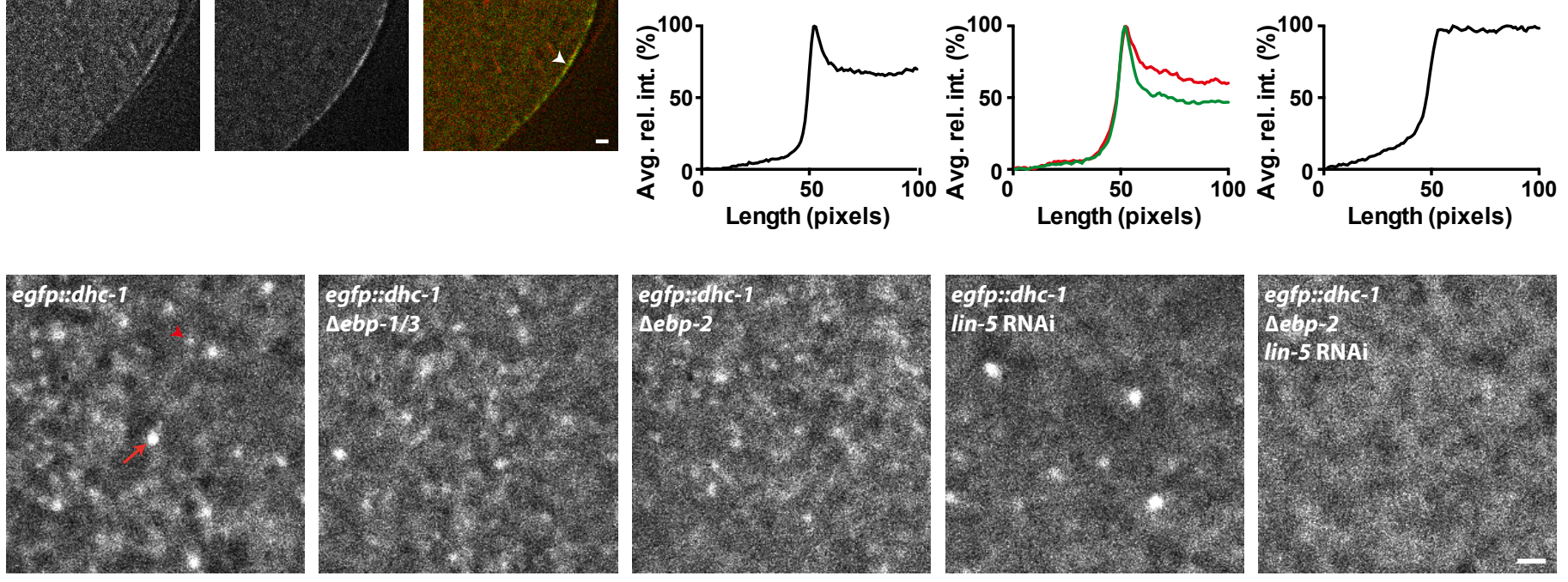
1 Figure 5. Loss of EBP-1/2/3 affects MT dynamics but does not affect mitotic

\section{2 pulling force generation.}

3 (A) Quantification of centrosome rocking during anaphase in wt and $\Delta e b p$

4 embryos. The max. amplitude is presented as a \% of embryo height for both the

5 anterior (green) and posterior (red) pole. Bars represent average $+\mathrm{SD},{ }^{*} \mathrm{P}<0.05$

6 compared to wt. Unpaired Welch Student's $t$-test. $N$-values are indicated below bar

7 graphs. (B) Example of spindle bisection using an ultraviolet laser (curved purple

8 line) in an embryo expressing GFP::TBB-2 (MTs). Five consecutive frames are shown,

9 each the average of 2 consecutive frames from a $500 \mathrm{~ms}$ exposure stream-lapse

10 movie. Arrows indicate the direction and arbitrary relative speed of displacement

11 upon spindle bisection. Anterior to the left. Scale bar, $1 \mu \mathrm{m}$. (C) Quantification of

12 average peak velocity ( $\mu \mathrm{m} / \mathrm{s}$ ) upon spindle bisection for anterior (green) and

13 posterior (red) centrosomes. Bars represent average + SD, no indication means no

14 significant difference from wt. Unpaired Welch Student's t-test. N-values are

15 indicated below bar graphs. (D) Quantification of cortical residence time of end-on

16 MT contacts as visualized by TIRF microscopy of one-cell embryos expressing either

17 GFP::TBB-2 or TBA-2::YFP during early anaphase (schematically represented on the

18 left), in different $\Delta e b p$ mutant backgrounds. Representative image shown is an

19 average of 5 consecutive frames from a 250 ms stream-lapse movie, after

20 background subtraction by a gaussian blur filter. Scale bar, $1 \mu \mathrm{m}$. Average cortical

21 residence time in seconds. Bars represent average $+\mathrm{SD}$, **** $\mathrm{P}<0.0001$ compared

22 to wt, ns not significant. Unpaired Welch Student's $t$-test. N=100 tracks for each 
bioRxiv preprint doi: https://doi.org/10.1101/118935; this version posted March 21, 2017. The copyright holder for this preprint (which was

not certified by peer review) is the author/funder, who has granted bioRxiv a license to display the preprint in perpetuity. It is made available under aCC-BY-NC-ND 4.0 International license.

1 condition. (E) Model of force generation in wt (left) and $\Delta e b p-1 / 2 / 3$ (right) as

2 explained in the text. 
Fiq. 5 bioRxiv preprint doi: https://doi.org/10.1101/118935; this version posted March 21, 2017. The copyright holder for this preprint (which was not certified by peer review) is the author/funder, who has granted bioRxiv a license to display the preprint in perpetuity. It is made available under aCC-BY-NC-ND 4.0 International license.

A

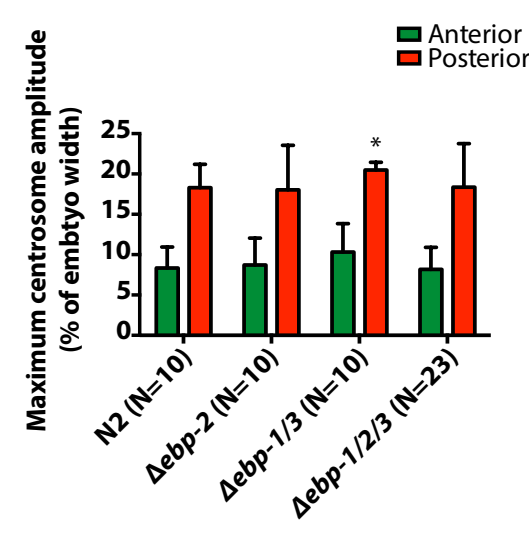

B

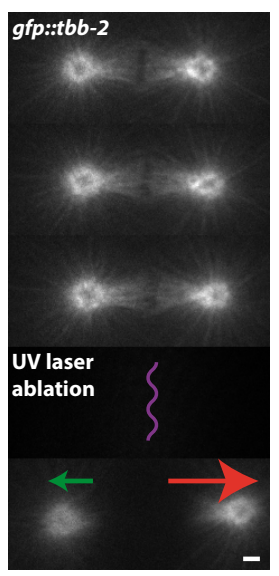

C

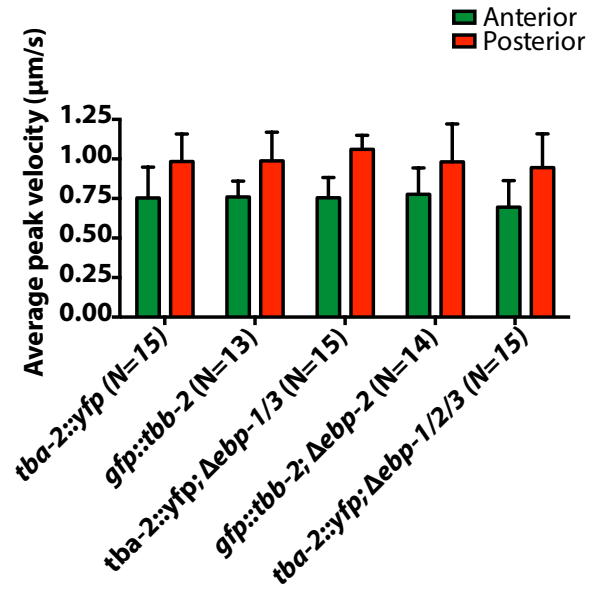

D
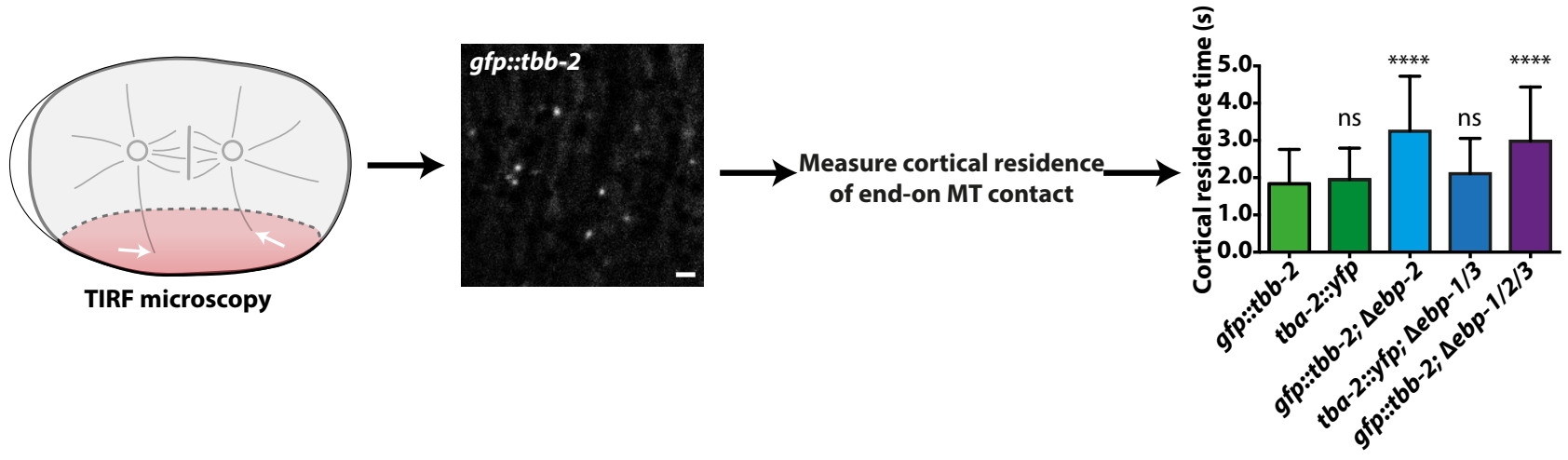

E

Wild type

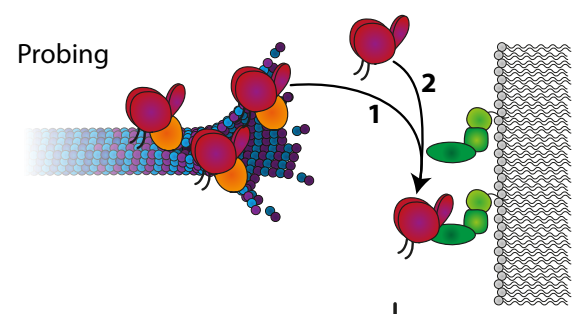

Force generation

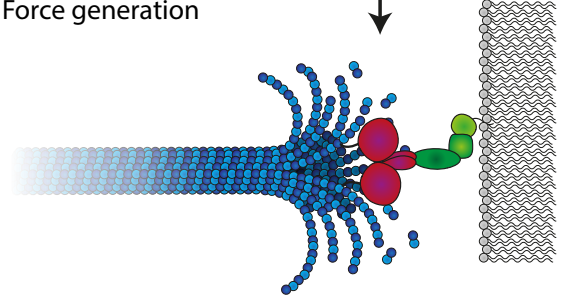

$\Delta e b p-1 / 2 / 3$

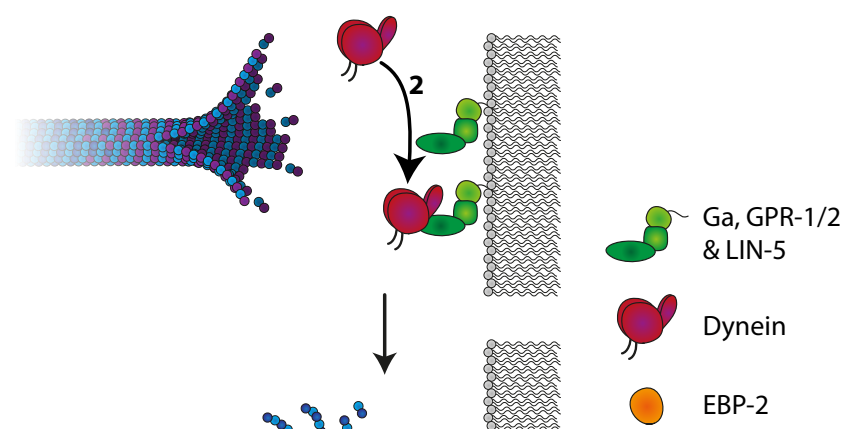

